\title{
VI.
}

Aus dem hirnanatomischen Laboratorium des hauptstädt. Elisabeth-Siechenhauses.

\section{Beiträge zur Nosographie und Histopathologie der amaurotisch-paralytischen Idiotieformen.}

\author{
Von \\ Prof. Karl Schaffer \\ in Budapest. \\ (Hierzu Tafel IV und V und eine Abbildung im Text.)
}

Mit Recht sagt Kraepelin ${ }^{1}$, dass durch das Wirrsal der idiotischen Krankheitszustände als Führer uns nar die pathologische Anatomie dienen kann. Hierbei bezieht er sich auf den, in dieser Beziehung sicherlich: erfahrensten Bourneville, der auf pathologisch-anatomischer Gruudlag den Idiotismus in folgende Formen zergliedert ${ }^{2}$ ): 1. Idiotismus durch Hydrocephalie, 2. Idiotismus durch Mikrocephalie, 3. Idiotismus durch Entwickelungshemmung der Hirnwindungen, 4. Idiotismus durch einen Bildungsfehler des Gehirns, a) angeborener (Porencephalie, Fehlen des.Balkens), b) pathologischer (Pseudocysten, gelbe Erweichungsherde, Pseudoporencephalie); 5. Idiotismus durch hypertrophische Hirnsklerose, 6. Idiotismus durch atrophische Sklerose (einer oder zwei Hemisphärren, eines Lappens, einzelner Hirnwindungen); 7. Idiotismus bei Meningitis oder Meningoencephalitis, 8. Idiotismus mit Pachydermie, 9. Idiotismus bei Hirntumoren, 10. Traumatischer Idiotismus.

Trotz der grossen Wichtigkeit der patbologischen Anatomie in der Aufstellung von einzelnen Formen, drängten gewisse klinisch schärfer markirte Formen ohne Rücksicht auf die pathologische Anatomie resp.

1) Klinische Psychiatrie. 1904. S. 871.

2) Siehe Kaes' Referat: Neuere Arbeiten zur patholog. Anatomie der Idiotie. Monatsschr. f. Psych. u. Neur. 1897. S. 496. 
Histopathologie zur Bestimmung gewisser Idiotiearten. Namentlich war die von Warren-Tay und Sachs genau geschilderte amaurotischparalytische familiäre Idiotie eine solche scharf umschriebene Form und in neuester Zeit machte Heinrich Vogt in einer sehr klar geschriebenen Arbeit ${ }^{1}$ ) den Versuch, innerhalb des Sammelbegriffes der Idiotie die Sachs'sche Form sammt verwandten Krankheitsbildern als einen gemeinsamen und wohlumschriebenen Typus abzugrenzen. Die grundlegenden Erscheinungen dieses Vogt'schen gemeinsamen Typus sind nach Angabe dieses Autor's ${ }^{2}$ ) folgende: 1. Schwäche der Extremitäten bis zur völligen Lähmung meist diplegischer Art, selten paraplegisch; Lähmung bald spastisch, bald schlaff. 2. Abnahme der Sehkraft bis zur völligen Blindheit; ophthalmoskopisch lässt sich Sehnervenatrophie feststellen, zu welcher in den Sachs'schen Fällen noch der bekannte, äusserst charakteristische Maculabefund kommt. 3. Psychischer Defect, mit der Zeit zur völligen Verblödung führend. 4. Rückschritt der körperlichen Entwickelung, Stillstand des. Wachsthums, Verdauungsstörungen, Marasmus, Tod durch Erschöpfung. 5. Fortschreitender Charakter der Erkrankung, zu welcher sich als secundäre Erscheinungen Anosmie, Taubheit, bulbäre Symptome, Coordinationsstörungen gesellen. 6. Familiarität des Leidens.

Der also charakterisirte Typus lässt nach H. Vogt zwei Gruppen unterscheiden. In die I. Gruppe gebört die Sachs'sche Form, für welche ausser dem bereits erwähnten Maculabefund noch der Umstand bezeichnend ist, dass die Affection frühzeitig, im Säuglingsalter oder im ersten oder zweiten Lebensjahre beginnt, somit handelt es sich hier nach Vogt um den Verlust der Bildungsfähigkeit, um Stillstand und Rückgang in der geistigen Entwickelung. In die II. Gruppe rechnet Vogt Fälle, in welchen die Erkrankung Individuen vom 4 . bis zum 16. Jahre befällt; auch hier, wie in den Sachs'schen Fällen, zeigt sich eine familiär auftretende Verblödung, welcher die Blindheit zumeist voraneilt. Letztere Erscheinung, ophthalmoskopisch Opticusatrophie, ]ässt den Sachs'schen Maculabefund constant vermissen; beginnt langsam und schreitet allmälig fort und wird zunehmend eine vollständige. Vogt erblickt im laculabefund der Sachs'schen Fälle kein differenzirendes Moment, da nach seiner Bemerkung dieses Symptom nach neueren Beobachtungen nicht bei allen Sachs'schen Fällen vorhanden ist. Es gäbe also nach Vogt auch eine Sachs'sehe familiär-amauro-

1) Ueber familiäre amaurotische Idiotie und verwandte Krankheitsbilder. Monatsschr. f. Psych. u. Neurol. XVIII. H. 2.

2) 1. c. S. $336,337$. 
tische Idiotie ohne Maculabefund; als eigentliches Unterscheidungsmoment zwischen den zwei Vogt'schen Gruppen könne nur das Alter dienen, obschon auch hier Uebergänge beobachtet wurden (Fall von Koller, welcher einen Sachs'schen Kranken betraf, der zur Zeit der Beobachtung, fünf Jahre alt, noch lebte). Dieser fliessenden Uebergänge ungeachtet, lassen sich nach $\mathrm{Vogt}$ die familiär-amaurotischen Idiotien in zwei Gruppen trennen, deren erste von "infantilem Charakter" die typischen Sachs'schen Fälle enthält, deren zweite von „juvenilem Charakter" auf die familiär amaurotischen Idiotien des Jünglingsalters sich bezieht. Das typische Krankheitsbild dieser Gruppe juvenilen Charakters schildert Vogt wie folgt: Mehrere bis dahin gesunde Kinder einer Familie (scheinbar ohne Rassendisposition, entgegen den Sachsschen Fällen, welche sich überwiegend auf jüdische Kinder beziehen) erkranken während des schulpflichtigen Alters und zwar mit Abnahme der Sehkraft, welche nach Honaten zu völliger Erblindung führt. Nun folgt Abnahme der geistigen Regsamkeit; die bereits erlernten Fähigkeiten (Lesen, Schreiben, Sprache) verlieren sich. Die Kranken werden unrein, apathisch, kennen schliesslich ihre eigene Mutter nicht. Zuletzt tritt die motorische Schwäche auf, welche bald schlaff, bald spastisch ist. Das terminale Stadium ist ein Zustand von völliger Reactionslosigkeit; die Kranken sind monate-, selbst jahrelang moribund, ehe der Tod eintritt.

H. Vogt gruppirt in seiner Arbeit die übereinstimmenden und die abweichenden Züge der infantilen und juvenilen Gruppe der familiäramaurotischen Idiotien. Als übereinstimmend erweist sich die Familiarität, ferner die Symptomatologie (Blindheit, Lähmung, Verblödung; schliesslich der exquisit progrediente Verlauf). Die divergirenden Züge sind: 1. Die Bevorzugung der jüdischen Rasse durch die Sachssche Idiotie; 2. der Macularbefund, welcher bei der juvenilen Form nie vorkommt, hingegen die Sachs'schen Fälle durchwegs charakterisirt; 3. das Einsetzen des Leidens in verschiedenem Lebensalter, die infantile Form im ersten Lebensalter und endet im dritten Jahre, während die juvenile Form im vierten Jahre erst beginnt. Vogt weist jedoch darauf hin, dass die trennenden Momente "nur die Modification eines einheitlichen Typus" wären, somit seien die Sachs'sche Form und die Fälle der juvenilen amaurotischen Idiotie „Repräsentanten einer gemeinsamen einheitlichen Krankheitsgruppe". Desshalb betont auch Vogt, dass zwischen beiden Gruppen nach Alter und Verlaufsart Uebergänge existiren. "Weseutlich ist, dass die Kinder erst normal sind, das Leiden beginnt nie von Geburt an. Die Sachs'sche Gruppe. stellt sich als die intensivere Form dar, einmal insofern sie in früherem Alter ein- 
setzt (das Gehirn versagt vorher), zweitens insofern der Verlauf mehr foudroyant ist." Speciell den Maculabefund, welcher von allen Autoren klinisch für die Sachs'sche Form charakteristisch bezeichnet wird, erachtet Vogt nicht als eine conditio sine qua non, wobei er sich auf die Fälle von Mülberger, Heveroch und Koller bezieht, in welchen zwar atrophische Papillen zugegen waren, jedoch keine Maculaveränderung sich vorfand.

Interessant ist es, dass $\mathrm{H}$. Vogt sich bezüglich der Pathogenese der familiär-amaurotischen Idiotien auf denselben Standpunkt versetzt, welchen Edinger und ich eimnahmen, d. h. er reiht diese Erkrankungen in die von Edinger statuirten "Aufbrauchkrankheiten" indem er dieselben durch "allgemeines Versagen des Cerebrums" charakterisirt.

Soweit H. Vogt über die familiär-amaurotischen Idiotien. Es sei mir nun gestattet, dass ich auf Grund meiner klinisch-histologischen Erfahrungen zur Sache Einiges beisteuere.

H. Vogl's klinische Erörterungen (seine anatomischen Untersuchungen werden später erscheinen) gipfeln in dem Satz, dass die (Sachs'sche) infantile und die juvenile Form der familiär-amaurotischen Idiotie eigentlich einen gemeinsamen Typus darstellen. So sehr diese Behauptung: unserem Bedürfniss der Verallgemeinerung entspricht, so ist dieselbe selbstverständlich nur anf Grund einer histopathologischen Prüfung zu acceptiren oder abzulehnen. $O b$ die zwei Formen H. Vogt's nur Modificationen innerhalb eines Typus darstellen, ist mangels anatomischer Charakterisirung schliesslich doch nur eine subjective Auffassung. Bezüglich der Bedeutung der Maculaveränderung als differenzirendes klinisches Charakteristikum vermag ich mich über dasselbe nicht ohne weiteres hinwegsetzen, besonders angesichts der Thatsache, dass von 86 Fällen nur in fünf dieser Befund vermisst wurde (s. oben). Doch selbst diese kleine Zahl ist zu reduciren, da von den negativen Fällen in einem Heveroch's, welcher zur Section kam, makroskopisch eine auffällige Derbheit des Gehirns zu constatiren war, namentlich aber waren die hinteren Theile der Hemispbären entschieden kleiner als die vorderen. Da ich in sieben Fällen voll Sachs'scher Form, welche alle zur Section gelangten, in keinem einzigen eine derartige, ja überhaupt keine makroskopisch wahrnehmbare Veränderung sah, so denke ich in begründeter Weise zu vermuthen, dass dieser Fall Heveroch's eigentlich eine lobäre, vielleicht eine Hemispärensklerose darstellt, und als solcher mit dem histopathologischen Wesen der Sachs'schen Form absolut nicht übereinstimmt. Nachdem nun der zweite, zur Zeit der Publication noch nicht zur Section gelangte Fall Heveroch's die Schwester des soeben besprochenen Falles war, so glaube ich - ange- 
sichts des hier ebenfalls mangelnden Maculabefundes - nicht ohne Grund anzunehmen, dass derselbe gleichfalls keine Sachs'sche Form war, wodann im Ganzen nur drei Fälle von angeblich Sachs'scher Idiotie mit negativem Maculabefund bleiben. Ob diese drei Fälle (gegen 78) genügen, um eine Form von Sachs'scher Idiotie ohne Maculabefund aufzustellen, mag dahingestellt sein.

Es ist und bleibt eine klinisch bemerkenswerthe Thatsache, auf welche H. Vogt zuerst mit Nachdruck hinwies, dass aus dem grossen Gebiete der Idiotie eine gewisse Anzahl von Patienten, welche durch die Familiarität, Blindheit, Lähmung (nebst der selbstverständlichen Idiotie) sich auszeichnen, abzusondern sind, welche alsdann eine nicht geringe klin is ch e Gruppe, die familiäre amaurotisch-paralytische Idiotie, bildet. Dieser klinische Begriff präjudieirt aber natürlich absolut nicht der Pathohistologie dieser klinischen Gruppe und es sei mir in diesen Zeilen gestattet, auf die Thatsache scharf hinzuweisen, wie ungemein die verschiedenen Fälle der amaurotisch-paralytischen Idiotie von einauder anatomisch, daher ihrem Wesen nach differiren. In zwei Arbeiten ${ }^{1}$ ) über die Histopathologie der Tay-Sachs'schen amaurotischen Idiotie wies ich nach, dass dieser Idiotieform eine ungemein scharf charakterisirte Erkrankungsform der Nervenzellen, namentlich jener der Hirnrinde entspricht, welche meines Wissens bisher bei anderen Erkrankungen des Centralnervensystems nicht erhoben wurde. Wennschon die prägnantesten Zellveränderungen in der Grosshirnrinde gefunden wurden, so waren diese in etwas schwächerem Grade auch an den übrigen Nervenzellen der gesammten graueh Substanz des Centralnervensystems aufzufinden; diese gaben sich in einer Zunahme der Interfibrillärsubstanz, somit in einer Schwellung des Zellkörpers resp. der Dentriten kund, wodurch des von mir als "cystische Degeneration" bezeichnete Bild entstand. Bezüglich der Einzelheiten dieser Zellentartung verweise ich auf meine erwähnten Arbeiten. Hier möge nur noch die fundamentale Thatsache hervorgehoben sein, dass grob-makroskopische Veränderungen, wie etwa in einem Fall Heveroch's, in keinem meiner sieben Fälle vorkamen. Ich definirte anatomisch die Sachs'sche amaurotische Idiotie als ein, in seinen nervenzelligen Elementen minderwerthiges Centralnervensystem, welches durch ungemein rasche Abnützung des nicht lebensfähigen Nervenzellprotoplasmas eine totale, auf die ge-

1) a) Zur Pathogenese der Tay-Sachs'schen amaurotischen Idiotie. Neurol. Centralbl. 1905. No. 9 und 10. - b) Weitere Beiträge zur pathologischen Histologie der familiären amaurotischen Idiotie. Journ. f. Psych. und Neurol. Bd. VI. Heft 1, 2. 
sammte graue Substanz sich ausdehnende Entartung erleidet. Congenitale, makroskopische Anomalien, Bildungsfehler spielen in der Pathogenese der Sach s'schen amaurotischen Idiotie keine Rolle; diese Idiotieform ist eine mikroskopisch scharf charakterisirte Art. Es ist nur natürlich, dass wir für alle, als Sachs'sche Idiotien erklärten Fälle dasselbe, soeben angedeutete histologische Substrat erfordern.

Im Gegensatz hierzu, d. h. abweichend von diesem Bilde will ich nun über die Präparate eines Falles von amaurotisch-paralytischer Idiotie, jedoch vermutblich ohne Familiarität berichten, welcher als juvenile Form über ein ganz anderes anatomisch-pathologisches Subtrat verfügt als die Sachs'sche Form.

Katharina T. recip. im Juli 1892 in das Elisabeth-Siechenhaus, war im Jahre 1895, als ich ihren Status aufnahm, 19 Jahre alt. Mangels nächster Anverwandter (Eltern längst verstorben), ist anamnestisch nichts festzustellen.

Status praesens. Stirn schmal; Antlitzknochen entwickelt, besonders stark der Unterkiefer. Zähne defect. Morel-Ohren. Schädelmaasse: Sagittaldurchmesser $163 \mathrm{~mm}$, Biparietaldurchmesser $120 \mathrm{~mm}$, Bitemporaldurchmesser $100 \mathrm{~mm}$, Höhendurchmesser $126 \mathrm{~mm}$, Circumferenz $485 \mathrm{~mm}$.-Pupillen mittelweit, gleich gross, reagiren auf Licht gehörig. Augenbewegungen frei, Strabismus. Linker Mundfacialis scheint energischer zu functioniren. Zungenbewegung unbehindert; Schlucken frei. Die Haut zeigt an den distalen Extremitätsabsehnitten eine röthlich-cyanotische Verfärbung. Skelet mittelmässig entwickelt; Rückgrat kyphoskoliotisch nach rechts. Thyroidea nicht palpabel. Die äusseren Genitalien stark entwickelt; Mammae gross mit starker Warze. Stuhl und Urin lässt Patientin unter sich. - Die Kranke liegt beständig im Bett mit froschartig hinaufgezogenen Füssen. Wird sie anfgesetzt, so sinkt der Rumpf sammt Kopf nach vorn (Fig. 1). Der Oberarm wird stark adducirt, der Unteram rechtwinkelig eingebogen gehalten; Oberschenkel dem Bauche genähert, legt sich dem höchstgradig im Knje eingebogenen Unterschenkel eng: an. Die Muskulatur ist in ihrer Entwickelung gehemmt. Die Sternocleidomastoidei erscheinen als dünne Stränge: Cucullaris und Nackenmuskeln bypertonisch. An der Oberextremität ist der Biceps nur federkielstark, der Triceps äusserst dünn und flach. Der Unterarm besitzt eine in toto hochgradig zurückgebliebene Muskulatur, während die kleinen Handmuskeln relativ besser entwickelt sind. Glutaei schwach, ebenso die ganze untere Extremität. Oberextremität weist starke Flexionscontractur auf; der Spasmus der Unterextremität ist womöglich noch bedeutender. Unterschenkel ist nur zu $90^{\circ}$ bei Aufwand von grosser Kraft zu strecken. Die Zehen befinden sich in Plantarflexion, der Fuss ist in Hackenstellung. Circumferenz der Extremitäten: Oberarm $15 \mathrm{~cm}$, Unterarm $12 \mathrm{~cm}$; Länge des Oberarms $29 \mathrm{~cm}$, des Unterarms $21 \mathrm{~cm}$, Schenkelumfang im mittleren Drittel $28 \mathrm{~cm}$, Unterschenkel $16 \mathrm{~cm}$. Länge des Oberschenkels (Spina 0ss. il. ant. sup. - Capit. fibulae) $37 \mathrm{~cm}$, des Unterschenkels (Capit. fib. - Mall. ext.) $25 \mathrm{~cm}$. 
Tricepsreflex und Kniephänomen auslösbar; sie sind lebhaft, jedoch der Contractur halber von kleinem Ausschlag. Muskelkraft kann nicht geprüft werden. Hautempfindlichleit scheint anf dem ganzen Körper fehlerlos zu

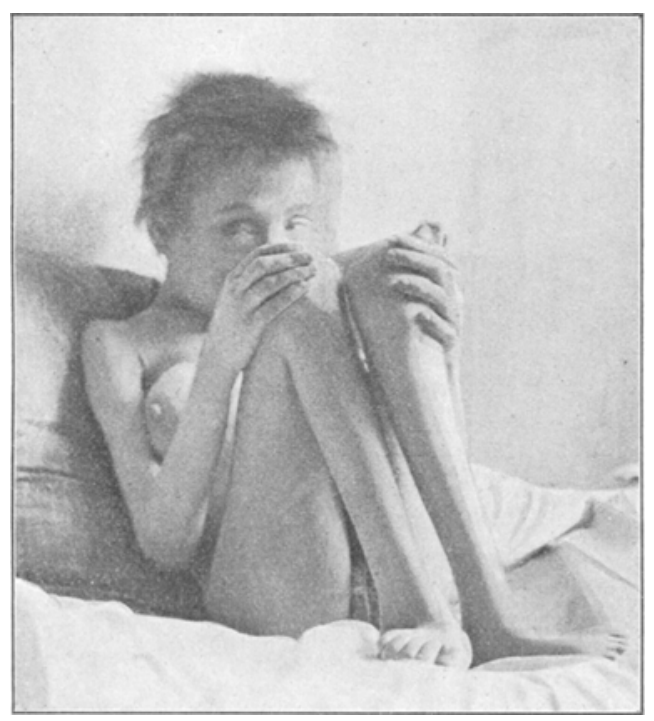

Figur 1.

sein; doch ist es auffällig, dass bei Anwendung schmerzhafter Reize (Stiche) Patientin zwar reagirt, ihre Mimik scheint aber hierbei keinen Schmerz auszudrücken, auch macht sie keine Abwehrbewegungen.

Sehkraft erscheint höchstgradig geschwächt (ophthalmoskopisch leider nicht untersucht) erkennt Personen nur durch deren Stimme.

Gehör gut. - Kranke leidet an häufigen epileptischen Anfällen mit Zungenbiss und Urinincontinenz.

Status mentalis. Auf Fragen reagirt sie nicht. Zumeist starrt sie mit leblosen Augen vor sich hin, lacht hin und wieder unmotivirt, und ist häufg mit dem Abtasten der Brüste beschäftigt. Sprache beschränkt sich nur auf einige Worte oder Wortrudimente, welche zumeist fehlerhaft ausgesprochen werden; z. B. "Pa Tante!, "Tjuppe" (statt Suppe), "Vode" (statt Wasser". Ihre Bettnachbarin, eine Tabikerin, hat ihr einige Worte eingelernt, deren Sinn sie verstand. Von selbst äusserte sie nie einen Wunsch; frug man sie: Bist du hungrig? so antwortete sie entweder „hm" (gleichbedeutend mit ja) oder "nein". Am liebsten spielte sie mit einem Kranz von leeren Zwirnspulen, welchen sie "Kuli" nannte; fand sie diesen nicht sogleich, so schrie sie "mein Kuli". - Wollte sie Jemandem drohen, so sagte' sie "Wart" Tant Marie"; 
Dieser Satz bezog sich auf eine Nachbarin, welchen sie oft hörend, mechanisch wiedergab. Des Morgens sagte sie zur Wärterin „Mama fünf Uj" (Uhr); dies ist nämlich im Sommer die Zeit des Aufstehens. - Menstruirt hat sie nie.

Patientin starb im Juli 1900 im Alter von 24 Jahren in Folge einer Pneumonie. Das Gehirn zeigte makroskopisch nur eine anscheinend derbere Consistenz in toto; sonst war aber nichts Auffallendes zu finden. Ich zerlegte das Centralnervensystem in Serienschnitte, und zwar die eine Hälfte in Frontal-, die andere in Horizontalschnitte, deren Beschreibung ich im folgenden gebe. Färbung nach Weigert-Wolters.

\section{Froutalschnitte (Tafel IV).}

Fig. 1 entspricht dem vordersten Ende des Schwanzkernes (NC). Auf den ersten Blick fällt der ungemein grosse Seitenventrikel (VI) auf, als Ursache hierfür ist sofort der Mangel einer centralen weissen Markmasse zu erkennen. Sämmtliche Windungen $\left(\mathrm{L}=\mathrm{g}\right.$. fornicatus, $\mathrm{F}_{1},,_{2}, 3$ $=$ Stirnwindungen; $\mathrm{Fb}=$ basale Stirnwindungen, $\mathrm{sr}=$ sulc. rectus, $\mathrm{T}_{2}$ $=g$. temporalis sec.) weisen zwar ein ausgeprägtes Windungsmark auf; auch sieht man z. B. zwischen $\vec{F}_{1}$ und $\mathrm{F}_{2}$ in distinctester Weise die Windungsassociation (U-Fasern), doch fehlt die compacte centrale Marksubstanz. Alle Windungen sitzen auf einem zumeist recht dünnen Markstiel; auch ist es bemerkenswerth, dass im Centrum der meisten Windungen ein gliöser und gefässtragender Spalt sich befindet, wodurch das Windungsmark in eine rechte und in eine linke Hälfte getheilt wird; jede Hälfte ziebt dann mit ihrer Markmenge zur benachbarten Windung. Dieser Spalt ist besonders in $\mathrm{F}_{3}$ und $\mathrm{F}_{2}$ klar ausgeprägt. Die Windungen werden gegen den ausgehöhlten Ventrikel zu mit einer markhaltigen Membran abgegrenzt. Zwischen den Schwanzkern- und den basalen Frontalwindungen ist eine centrale Markmasse, welche den Stabkranz des Stirnlappens enthält, sichtbar. Besondere Beachtung verdient der Balken (Ce). Dieser erscheint bier in zwei Formationen; Cc repräsentirt das vorderste Ende des Balkenkörpers in der Form eines sehr dünnen Streifens, dessen Fasern hauptsächlich in $F_{1}$ übergehen. $\mathrm{Cc}_{1}$ entspricht dem Balkenknie und erscheint als Forceps anterior besonders im Verhältniss zu Cc nicht schwach entwickelt.

Fig. 2 entspricht einer frontalen Ebene durch das vorderste Ende des Sehhügels. Auch hier, wie überhaupt an allen folgenden Schnitten fällt die Ventrikelektasie auf den ersten Blick auf. Balken (Cc) ist hier ebenfalls ein dünner Streifen von markhaltigen Fasern. Die erste Frontalwindung, einen centralen Spalt enthaltend, sitzt ebenfalls einem auffallend schmächtigen Stiel auf, entbehrt somit hier noch eine centrale Markmasse. Doch bemerkt man schon hier ein dünnes Strängchen von Markfasern, welche den Vl als ein concav geformtes Bündelchen 
umkreisend, der $F_{1}$ zustreben ( $C R=$ corona radiata); Ursprung dieser Fasern liegt in der inneren Kapsel (zwischen $\mathrm{NC}$ und $\mathrm{Nl}_{3}=$ Putamen), gehören daber zu den Projectionsfasern der Hemisphäre. Unterhalb der CR liegt ein dünner Saum, dessen feine, schwach gefärbte Markfasern in den oberflächlichen Saum des NC übergeht; es ist dies das, Markmasse führende Ventrikelependym. Es soll nicht unerwähnt bleiben, dass die Markmasse der $\mathrm{F}_{2}$ mit jener der vorderen Centralwindung (Ca) breit confluirt; letztere geht in eine wohl markirte äussere Kapsel, dem $\mathrm{N}]_{3}$ anliegend, über. Die Insel $(J)$ sowie die Schläfenwindungen zeigen eine relativ gute Entwickelung. Fli entspricht dem in den Hirnanatomien als Fasciculus longitudinalis inferior bezeichneten Faserzug, über dessen strittige Natur - ob Associationsbündel, ob Projectionsbündel — später noch einiges erwähnt werden soll. Mit besonderem Nachdruck möchte ich auf diesem Schnitt auf den Sehnerv (II) aufmerksam machen, welcher hier zum Chiasma nahe, als tadellos markhaltiges Bündel erscheint. $\mathrm{Th}=$ Sehhügel; $\mathrm{CF}=$ Fornixsäule $; \mathrm{Al}=$ ansa lenticularis; $\mathrm{U}=$ Uncus; $\mathrm{Am}=$ amygdala; ot $=$ sulc. occipitotemporalis.

An Fig. 3 erscheint die Ebene durch das vordere Drittel des Sehhügels wiedergegeben. Ausser der Ventrikelaushöhlung (Vl) fällt hier noch als neues Moment die Projectionfaserung (CR) als compacter, stärkerer Strang auf, dessen Entwickelung aus der inneren Kapsel (CJ) hier zweifellos ist; bemerkenswerth ist auch das Eindringen der CR in die $F_{1}$ und $F_{2}$. Der Balken (Cc) ist hier gleichfalls abnorm dünn und geht hauptsächlich in $F_{1}$ über. Hervorzuheben ist, dass an diesem Schnitte so die vordere (Ca) wie hintere Centralwindung (Cp) nur ein Windungsmark besitzen; dieser. Umstand ist besonders klar an Cp zn sehen. Unsere Aufmerksamkeit verdient noch vor Allem der ganz normale Sehtract (II); ferner der normal gebaute Sehhügel (Th), in welchem hier das Vicq d'Azyr'sche Bündel (VA) enthalten ist. Die CJ geht hier in einen normale Faserung aufweisenden Pedunculus (P) über. Fli $=$ unteres Längsbündel; VIs $=$ das Unterhorn des Seitenventrikels. Tth = taenia thalami. $\mathrm{F}=$ fornix.

Fig. 4 fällt etwa durch die Mitte des Sehhügels. In diesem Präparate zeigt die Ca ihre maximale Enwickelung; sie enthält ausser dem eigenen Windungsmark noch Projectionsfaserung (CR), welche aber in dieser Frontalebene bereits ziemlich reducirt erscbeint. Bemerkenswerth erscheint der Umstand, dass die Cp eine Einstrahlung von Projectionsfasern entbehrt. Neben der gut entwickelten Insel ist noch die erste Schläfewindung aus dem Grunde bemerkenswerth, weil ein Theil des Windungsmarkes dem $\mathrm{Nl}_{3}$ resp. sublenticulären Segment der inneren Kapsel zustrebt, wie dies besonders instructiv an den später anzuführen- 
den Horizontalschnitten sichtbar sein wird. Aus der sublenticulären Faserung verdient besonders die compacte Fasermasse W unsere Aufmerksamkeit; von derselben lässt sich einestheils unschwer nachweisen, dass sie rückwärts in das dreieckige Wernicke'sche Feld übergeht (siehe Fig. $5 \mathrm{~W}$ ), während sie nach vorne successive unterhalb des sogenannten Pedunculus des Putamen gelangt (s. Fig. $3 \mathrm{Fli}$ ) und somit zur Bildung jener frontalwärts convexen Schlinge beiträgt, welche Dejerine als Arnold'sches temporo-thalamisches Bündel (,faisceau temporo-thalamique) nenut. $\mathrm{CNC}=$ cauda nuclei caudati; Vls $=$ Unterhorn; $\mathrm{CA}=$ cornu Ammonis; II $=\mathrm{n}$. opticus; $\mathrm{P}=$ pedunculus; $\mathrm{Th}=$ Sehhügel; $\mathrm{Tth}=$ taenia thalami; $\mathrm{F}=$ fornix; $\mathrm{Ce}=$ Balken; $\mathrm{L}=\mathrm{g}$. fornicatus. Fus $=$ g. fusiformis.

Fig. 5 stellt einen Frontalschnitt durch das Pulvinar dar. Die einzelnen Formationen des Zwischenbirns sind der rothe Kern ( $\mathrm{Nr}$ ), das Ganglion habenulae (GH), das Meynert'sche Bündel (M), das Centre médian (NL), die Soemmering'sche Substanz (SS), der Pedunculus (P), das Corpus geniculatum laterale (cgl), an dessen oberen und äusseren Seite ein compactes Markfeld (W) das Wernicke'sche Feld zu sehen ist. Dieses Feld rückt in den mehr proximalen Ebenen successive mehr seit- und abwärts in die sublenticuläre Region (s. Fig. $4 \mathrm{~W}$ ). $\mathrm{NC}=$ nucleus caudatus; III $=\mathrm{n}$. oculomotorius. Der Markgehalt der soeben erwähnten Formationen zeigt normale Verhältnisse. Allein auffallend ist ein Feld zwischen $\mathrm{W}$ und $\mathrm{Pu}$ (putamen) durch seine Faserarmuth; es ist dies bekanntlich jene Stelle, welche die zur optischen (Gratiolet'schen) Strahlung strebenden Fasern enthält und welche speciell an den später folgenden Horizontalschnitten noch evidenter zum Vorschein gelangen wird. - Der Seitenventrikel (Vl) fängt an hier noch grösser zu werden. Die CR ist nur mehr in der Form eines schmächtigen Zuges sichtbar, der Balken erscheint hier wieder als ein dünnes Bändchen, welches allein zur Ca Beziehungen unterhält. Bemerkenswerth ist der Umstand, dass $\mathrm{Cp}$ keine Projectionsfaserung enthält, ferner die Thatsache, dass das untere Längsbündel (Fli) in dieser Schnittebene ganz auf den Grund des Unterhorns sich zurückgezogen hat und mit der sublenticulären Faserung bereits in keiner Beziehnng steht.

Fig. 6 ist das Bild eines Schnittes, welcher durch die hintere Commissur (Cop) geht. $\mathrm{Pa}=$ pulvinar, $\mathrm{Cgm}=$ corp. geniculat. mediale. Auf diesem Präparat ist die Confluenz des Seitenventrikels mit dem Unterhorn in der Form einer abnorm grossen Höhle (CVl) sichtbar, ebenso ist der Uebergang der Fimbria in den Fornixschenkel (F) und dessen Vereinigung mit dem Balken bemerkbar. Sämmtliche Windungen dieses Schnittes: $\mathrm{Ca}, \mathrm{Cp}$, unteres Scheitelläppchen $(\mathrm{Pi}), \mathrm{T}_{1},{ }_{2},{ }_{3}, \mathrm{Fus}$, 
entbehren eine centrale Markmasse. Im Pedunculus (P) ist dessen normale Markfaserung auffallend.

Fig. 7 führt uns dieselben Verhältnisse vor unsere Augen wie die bevorstehende; eine speciellere Beachtung verdient hier nur das Fli, welches ganz auf den Grund des Seitenventrikels (CVI) hinabgedrängt erscheint und aufwärts entlang der äusseren Wand des CVl keine Fortsetzung besitzt. Diese Stelle, nämlich die äussere Ventrikelwand ist höchst bemerkenswertb, denn wir vermissen hier vor Allem die optische Strahlung; auch das Tapetum (Tp) ist nur durch einen höchst dünnen, rudimentären $\mathrm{Zug}$ vertreten. Ferner fehlt hier in den höheren Etagen des Ventrikels, in der Höhe des Balkens der sonst so kräftig entwickelte obere Theil des Tapetum beim Uebergang in das Splenium. An dem der Hemisphäre anliegenden Vierhügel ist besonders die ganz normale Pyramidenfaserung bemerkbar.

Endlich wiedergiebt Fig. 8 einen Frontalschnitt durch den Hinterhauptslappen. Was hier ebenfalls auf den ersten Blick auffällt, das ist der totale Mangel der centralen Markfaserung des Occipitallappens; man findet hier keinen Forceps corporis callosi, keinen Fasciculus verticalis (Wernicke), keinen Fase. transversus cunei und vor Allem keine Sehstrahlung; einzig allein das untere Längsbündel (Fli) ist hier am Grunde des Cuneus als ein dreieckiges Fascikel sichtbar, dessen Uebergang in die analogen Stellen der bereits beschriebenen Schnittebenen leicht zu verfolgen ist. Eine specielle Erwähnung verdient hier die Calcarinarinde, deren Markreichthum an nicht allzustark differenzirten Schnitten den analogen Verhältnissen eines erwachsenen Menschen zwar nicht ganz gleichkommt, jedoch sei hervorgehoben, dass sämmtliche Faserschichten, besonders der bekannte Gennari'sche (alias Viq d'A zyr'sche) Streifen, sowie der dichte Zonalplexus eine gute Entwickelung zeigen. Noch wäre zu bemerken, dass eben in Folge des Mangels einer centralen Markmasse sämtliche Windungen dieses Schnittes nur ein Windungsmark besitzen und allein mittelst desselben mit den benachbarten Windungen in Verbindung stehen.

Fassen wir nun die Hauptzüge obiger Schilderung zusammen, so lassen sich an den Frontalschnitten eines Falles von amaurotisch-paralytischer Idiotie folgende morphologische Einzelheiten feststellen:

1. Bei normaler äusserer Configuration der Hemisphären ist der generelle Mangel an Associationssystemen des Vorderhirns auffallend, wodureh die centrale Markmasse des Grosshirns in auffallender Weise reducirt erscheint. So fehlt z. B. das Bogenbüschel (Fasc. arcuatus), das fronto-occipitale Bündel, um nur die auffallendsten feblenden Verbindungszüge zwischen den einzelnen Rindenbezirken zu nennen. Von den 
Commissurenfasern des Grosshirns ist der Balken in entschieden geschwächter Form vorhanden, worüber besonders nach Schilderung der Horizontalschnitte noch die Rede sein wird; an Frontalschnitten hat der Balken nur $1 / 3$ Dicke des normalen. Die Fornixcommissur soll an der Hand von Horizontalschnitten noch Erwähnung finden. Einzig der Stabkranz zeigt anuähernd normale Verhältnisse, doch giebt es auch in dieser Beziehung nicht unerhebliche Mängel. Relativ am stärksten präsentiren sich die Projectionszüge aus der inneren Kapsel resp. Sehhügel zu den Centralwindungen und umgekehrt; diese Verhältnisse geben sich einestheils in der normalen Markfaserung des Sebhügels (ventrolateraler Kern, s. Fig. 4), andertheils in der normalen Pyramidenfaserung kund; auch ist zu bemerken, dass die Rinde der Centralwindungen einen solchen Reichthum von Markfasern aufweist, welcher kaum jenem eine̊s normalen Individuums nachsteht.

Die aus dem Stimhirn, aus dem Hinterhauptslappen und aus dem Schläfenlappen hervorgehenden Projektionsfasern sollen auf Grund von Horizontalschnitten eingehender geschildert werden; hier sei" nur so viel bemerkt, dass sie nicht alle fehlen. Am meisten ins Auge springend ist die fehlende Sehstrahlung. Das Gewölbe erscheint zwar in schmächtiger Form, doch zeigt es keine wesentliche Anomalie. Ueber das sog. untere Längsbündel soll an der Hand von Horizontalschnitten noch eingehender gesprochen werden.

2. Durch die soeben geschilderte Reduction der centralen Markmassen entstehen Windungen, welche stilartig dem weissen Hemisphärencentrum aufsitzen, und welche nur ein Windungsmark sensu strictiori besitzen, wodurch die Verbindung mit benachbarten Windungen (Meynert's U-Fasern) bewerkstelligt wird.

3. Aus der mangelhaften Entwickelung der centralen Markmassen ist die colossale Ventrikelectasie leicht verständlich.

\section{Horizontalschnitte. (Tafel V.)}

Fig. 1 entspricht einem Schnitte, welcher knapp oberhalb des Balkens gelegt ist. F 1, 2, 3 sind die drei Stirnwindungen; $\mathrm{Ca}, \mathrm{Cp}$ die Centralwindungen, welche durch die Rolando'sche Furche $(\mathrm{R})$ getrennt sind; Pi ist das untere Scheitelläppchen; Om, s, entsprechen der mittleren und oberen Occipitalwindung; Pre ist der Praecuneus, endlich $\mathrm{L}=$ Gyr. fornicatus. Während all diese Rindenstellen normale Markfaserstructur zeigen, welche besonders schön an der vorderen Centralwindung sowie an der benachbarten dritten Frontalwindung erscheint, ist im Gegensatz hierzu ein sofort ins Auge fallender Mangel von centraler Markmasse zu bemerken. Allein im Niveau der Centralwindungen 
findet man von letzterer etwas, bezeichnet in der Abbildung mit $\mathrm{CR}=$ corona radiata; frontalwärts von dieser Stelle befindet sich ein faserleeres Gebiet zwischen der dritten Frontalwindung und dem Gyr. fornicatus, welches sich mit einem schmalen, marklosen Streifen zur Spitze des Stirnhirns zieht. Occipitalwärts von der Strahlenkrone zieht derselbe Spalt in der Markmasse des Hinterhauptlappens, doch giebt er bemerkenswerther Weise spornartige Fortsätze für das Windungsmark des unteren Scheitelläppchens und für den Praecuneus ab.

Der vollkommene Mangel an centraler Markmasse ist besonders deutlich und überzeugend an Fig. 2 sichtbar, welcher Schnitt die Bogenkuppe des Schwanzkerns trifft, zugleich aber den Balkenkörper passirt. Unsere Aufmerksamkeit fesselt auf den ersten Blick der enorm ausgeböhlte Seitenventrikel (Vl) und es ist unschwer zu erkennen, dass diese Ektasie eben durch den Mangel der centralen Hemisphärenmarkmasse entstand. Sämmtliche. Windungen dieses Niveaus besitzen aussehliesslich eine Eigenfaserung, wodurch guirlandartige Markverbindungen zwischen den benachbarten Windungen zu Stande kommen. Der somit auf Kosten der centralen Markmassen dilatirte Seitenventrikel wird an seiner lateralen Wand mit einer Ependymmembran eingesäumt, welche sich occipitalwärts als ein Ependymspalt (ep) in den Hinterhauptlappen fortsetzt, frontalwärts aber die Basis der Frontalwindungen besetzt, wobei diese Membran vom Kopfe des Schwanzkerns ausgehend, schwach gefärbte, dünne Markfasern fübrt (fnc, fasc., nuclei caudati), dann den Grund der Stirnwindungen umkreisend, unter den frontalen Theil des Gyr. fornicatus $\left(L^{\prime}\right)$ sich dem Balken so innig anschmiegt, dass sie hier schon nicht mehr differenzirt werden kann. Der Balken (Cc) erscheint als ein dünnes Band, welohes sich so frontal- wie occipitalwärts sehr rasch verschmächtigt. - CR ist die Corona radiata, welche an diesem Schnitte ausschliesslich unterhalb der Centralwindungen sich vorfindet; bemerkenswerth ist in dem Windungsmark der Ca und $\mathrm{Cp}$ ein gabelig getheilter Spalt, welcher aus einer faserleeren Gewebsspalte des Hemisphärencentrums (s. Fig. 1) entspringt. $I=$ insula, in deren Nachbarschaft eine mit zwei Sternen bezeichnete Windung durch ihre abnorme Faserarchitektonik auffällt. $\mathrm{S}=$ fossa Sylvii; $\mathrm{Pi}=$ lob. pariet. infer.; $0 \mathrm{~s}, \mathrm{~m}=$ =bere und mittlere Occipitalwindung; os $=$ sulc. occip. super.; po $=$ fiss. parieto-occipitalis; cllm $=$ sulc. calloso-marginalis.

Fig. 3 entspricht einem Schnitt, welcher die oberste Kuppe des Sehhügels (Th) getroffen hat. Durch letzteren wird die ungewöhnlich weite centrale Höhle (Vl) in zwei Unterabtheilungen getrennt; Vlf entspricht dem Vorderhorn, Vl ist die mittlere Partie des Seitenventrikels; CVl ist jener gemeinsame Theil des Seitenventrikels (Carrefour), aus 
welcher theils das Hinterhorn, theils das Unterhorn entspringt. Durch das Auftauchen der obersten Zacken des Putamen ist die Sonderung in eine innere und äussere Kapsel (Ci, Ce) ermöglicht: der Schwanzkern ist hier schon in zwei getrennten Theilen (Kopf $\mathrm{NC}$, Schwanz $\mathrm{NC}^{\prime}$ ) sichtbar; vom Kopftheil geht ein blasses Bündelchen fnc (fasc. nuclei caudati?) ab. Der Balken erscheint hier bereits in seinem hinteren Theil als Splenium $\left(\mathrm{Cc}^{\prime}\right)$ und vorderen Theil als Knie (Cc), zwischen welchen das Septum pellucidum (sp) sammt dem Ventriculus septi pellucidi ausgespannt ist. Aus dem Splenium zieht der Forceps major (fm) rückwärts und setzt sich als Tapetum ( ${ }^{t} \mathrm{p}$ ) an der medialen Ventrikelwand fort. F bedeutet das Gewölbe, welches nur insofern auffällt, dass es rom Balken resp. vom Splenium sich weit abhebt. Bemerkenswerth ist an diesem Schnitt die $\mathrm{Zwinge}(\mathrm{Cg})$, welche besonders in ihrem vorderen Theil deutlich erkennbar ist. $\mathrm{Cu}$ entspricht dem Cuneus; po ist die Fiss. parieto-occipitalis; ep der Ependymstreifen des Hinterhauptlappens; die Windungen stimmen mit jenen der Fig. 2 überein; neu hinzugekommen sind die obere und tiefe Schläfenwindung (Ts, Tp).

Fig. 4 ist die Reproduction eines Schnittes, welcher durch die grösste horizontale Ausdehnung des Sehhügels und durch das klassische Kapselknie führt. An dem Sehhügel ist hier der mediale und laterale Kern ( $\mathrm{nm}, \mathrm{nl}$ ) das Centrum medianum Luysii (NL) sowie die Habenula (H) erkennbar; am Pulvinar des Sehhügels fällt ein, durch seine Markleere ausgezeichnetes, helles Feld auf $(\mathrm{Pu})$, von welchem leicht festzustellen ist, dass es der Sehstrahlung zugehört. $\mathrm{Ne}^{\prime}$ ist der Schwanz, se entspricht der Stria cornea. Cip und Cia sind der hintere und vordere Kapselschenkel; beide erscheinen vollkommen normal bezüglich ihres Faserbaues. $\mathrm{Nl}_{3}$ und $\mathrm{Nl}_{1}+2$ sind die drei Glieder des Linsenkerns; am Aussenrand des Putamen $\left(\mathrm{Nl}_{3}\right)$ sieht man eine deutliche äussere Kapsel, welche von der Insel (I) durch einen dünnen Saum grauer Substanz, das Claustrum, getrennt ist. Ceg - genu corporis callosi; cle $=$ fissura calcarina; bezüglich der Windungen ist nur die zweite Temporalwindung $\left(T_{2}\right)$ neu hinzugekommen, ebenso das Ammonshorn (CA), welchem der Forceps minor (fi) sich ansetzt. Hinter letzterem sieht man ein schmächtiges Bündel, fli, im Marke des Hinterhauptlappens; es ist das untere Längsbündel. Vlo ist das Hinterhorn, welches hier von der bekannten Umsäumung mittelst Tapetum, unteres Längsbündel und Sehstrahlung nichts erkennen lässt. Bezüglich der Hirnwindungen ist an diesem Schnitte ebenso wie am vorhergehenden hervorzuheben, dass sie eine centralen Hemisphärenmarkmasse entbehren, besitzen daher nur eine Eigenfaserung, wie dies 
besonders auffallend am Stirnlappen erkenntlich ist; allein aus der ersten Temporalwindung geht ein stärkerer Zug von Markmasse gegen den retrolenticulären Abschnitt der inneren Kapsel (TK). Bei aufmerksamer Betrachtung der ersten Temporalwindung (Ti) fällt im Windungsmark ein feiner, lichter Streifen auf, welcher die Markmasse dieser Windung in zwei Theile trennt; der kieinere Theil geht als schmächtiges Markbündel zur zweiten Temporalwindung $\left(\boldsymbol{T}_{2}\right)$, repräsentirt als solches die Fibrae propriae der Rinde, während der grössere Theil, gestärkt durch einen mächtigen Zuzug aus der tiefen Temporalwindung (Tp) eine compacte Fasermasse bildet (TK), welche gegen das retrolenticuläre Segment der inneren Kapsel als das bekannte Türck'sche Bündel zieht, somit die temporale Kapselstrahlung repräsentirt. - Die Insel treffen wir hier in ihrer ganzen Ausdehnung; die Centralwindungen (Cp, Ca) erscheinen bereits reducirt.

Fig. 5 führt durch die Regio hypothalamica sowie durch den vorderen Vierhügel (Qa); Cop ist die hintere Commissur, NL = centrum medianum, FL entspricht dem Flechsig'schen schalenförmigen Körper hth ist das Haubenbündel des Sehhügels, hl entspricht dem Haubenbündel des Linsenkerns. Unsere Aufmerksamkeit verdient hier wiederum das Pulvinar $(\mathrm{Pu})$, welches faserleer erscheint. Ebenso erheischt eine specielle Würdigung die Umgebung des Unterhorns (VIs); die laterale Ventrikelwand besitzt gar keine normale Auskleidung mit dem bekannten Stratum sagittale occipitale und die daselbst befindlichen Windungen $\left(\mathrm{T}_{1}, \mathrm{~T}_{2}\right.$ ) verbinden sich einzig mit Eigenfasern; in das Marklager des Hinterhauptlappens dringt ein Ependymspalt (ep) hinein, gegen welchen zu aus dem Gyrus lingualis (lg) das untere Längsbündel (fli) zieht; fi entspricht dem Forceps minor. Beachtenswerth ist das Türcksche Bündel (TK), dessen Ursprung aus der ersten sowie tiefen Temporalwindung deutlich zu sehen ist. Alle übrigen Bezeichnungen sind schon bekannt. Ich möchte schliesslich noch darauf hinweisen, dass die Frontalwindungen in dieser Höhe noch keine centrale Markmasse besitzen.

Fig. 6 entspricht einem Schnitt, welcher durch eine tiefere Etage der Regio subthalamica, namentlich durch das Corpus subthalamicum (Lst) führt. Ich möchte hier besonders betonen, dass die gesammte Faserung dieser Gegend eine normale ist; sämmtliche Gebilde wie der rothe Kern (Nr), das Corp. subthalmicum, die beiden Corpora genieulata (gi, ge), der hier beginnende Hirnschenkel, der Linsen- und Schwanzkern zeigen gar keine Abweichung von der Norm. Nur die vordere Commissur (Coa) erscheint verhältnissmässig schwach. Worauf aber mit besonderem Nachdruck hingewiesen sei, das ist der äussere Kniehöcker 
(ge), welcher in seinem Markfaserbau ganz normale Verhältnisse aufweist, daher sich anders verhält wie das Pulvinar, von welchem wir auf Grund der Fig. 4 and 5 bereits erfuhren, dass dieses Gebild ganz markleer ist. Bemerkenswerth wäre ferner jenes kleine markreiche Feld, welches unmittelbar lateral dem äusseren Kniehöcker aufsitzt; dieses enhält die aus letzterem entspringende centrale Strahlung zum Hinterhauptlappen, ist daher als ein partielles Wernicke'sches Feld anzusehen, welchem nämlich der aus dem Pulvinar entspringende Zug abgeht. Es erübrigt mir nun die Aufmerksamkeit auf den Stirnlappen zu lenken, welcher in dieser Höhe bereits ein tiefes Mlark besitzt; man sieht ganz deutlich, wie der Rest der vorderen Kapsel aus dem Corpus striatum $\left(\mathrm{Nl}_{3}+\mathrm{NC}\right.$ ) herausbrechend, als frontale Strahlung (CRf) zum Pol des Stirnlappens zieht, um sich alsdann hauptsächlich in der ersten Stirnwindung zn verlieren. Im tiefen Mark des Stirnlappens fällt noch ein mil Ce bezeichnetes Feld auf, welches dem Balken entpricht. Unter der hier maximal entwickelten Insel $(J)$ zieht das distinct entwickelte Claustrum (CL); am Grund letzteren die äussere Kapsel (ce). Kehren wir zur hinteren Hälfte des Schnittes zurück; da sehen wir das Unterhorn an der medialen Wand von einem schmächtigen unteren Längsbündel (fi) eingesäumt, welches am hinteren Winkel des Unterhorns in ein äusserst schwächliches Bündelchen umbiegt, dessen Verlauf auf den Serienschnitton in der lateralen Ventrikelwand gegen das sublenticuläre Segment der inneren Kapsel zu verfolgen ist. Auf diesem Schnitte zeigt zuerst das Unterhorn eine annähernd normale Ausdehnung.

Fig. 7 wiedergiebt einen Schnitt, welcher durch die vordere durchlö cherte Platte (spa) führt. Wir bemerken hier den normalen Hirnschenkelfuss, welchem der tiefschwarz gefärbte, daher ganz intacte Sehnerv (II) als breites Band anliegt. Es sei betont, dass der Sehnerv so seines Markgehaltes, wie auch seiner Stärke nach von der Norm nicht im mindesten abweicht. Das Corpus mamillare (cm) erscheint bei mikroskopischer Betrachtung auch normal gebaut; es weist einen inneren, grösseren und einen kleineren, äusseren, faserreichen Kern auf. Das Stirnhirn besiizt eine gut entwickelte Strahlung (CRf); zwischen letzterem und Hirnschenkelfuss erstreckt sich die vordere durchlöcherte Platte (spa) und der basalste Theil des Putamen $\left(\mathrm{Nl}_{3}\right)$. Sulcus cruciatus ist mit er bezeichnet. Ausserhalb des starken Claustrum sieht man am Grund der Insel schwach gefärbte Züge des Fasciculus uncinatus (fu). Aus der ersten Temporalwindung zieht ein gut ausgeprägtes Tü rck'sches Bündel (TK) medial- und basalwärts. Einwärts davon erblickt man den Durchschnitt des sublenticulären Segmentes (Cisl) der inneren Kapsel. 
Das Unterhorn (Vls) wird durch das untere Längsbündel (fli) in einer Weise eingesäumt, welche dem Verhalten auf Figur. 6 vollkommen entspricht. - -

Schliesslich wäre Fig. 8 zu beschreiben; dieses Präparat ist der basalste Horizontalschnitt. Hier ist nur die Einsäumung des Unterhorns bemerkenswerth, welche durch zwei concentrisch parallel verlaufende Faserzüge gebildet wird. Das inuere Bündel kleidet die laterale Ventrikelwand aus; es dürfte dem Tapetum (tp) entsprechen. Das äussere Bündel als unteres Längsbündel (fli) zieht nach vorn und biegt lateral von Mandelkern (Am) aus der Sagittalrichtung in aufsteigende Richtung über (Cisl). Eine Verbindung zwischen Hinterhaupt- und Schläfelappen scheint das untere Längsbündel an diesem Präparat, wie denn überhaupt an allen übrigen Schnitten, nicht $z \mathfrak{u}$ bewerkstelligen. Am Stirnhirn ist mit rol die Riechwurzel bezeichnet; bemerkenswert erscheint hier noch der ganz normale Sehnerv (II). -

Die Durchforschung der Horizontalschnitte vorliegenden Falles ergab folgende Momente von allgemeiner Bedeutung.

Der Mangel an centralen Markmassen der Hemisphären ist auch an Horizontalschnitten auffallend, welcher Umstand klaffende, weit ausgehöhlte Innenräume, namentlich ein ungemein weites Vorderhorn bedingt. Die Abwesenheit des tiefen Markes macht sich besonders in dem Stirnlappen bemerkbar, besonders in dessen mittlerer und oberer Ebene, während die mehr basalen, vom unteren Theil des Schwanzkernkopfes beginnenden Ebenen bereits eine Projectionsstrahlungen besitzen (s. Fig. 6, Taf. V, CRf) als fronto-thalamische Verbindung. Eine zweite Projectionsstrahlung ist durch die thalamo-corticale sensible; sowie durch die motorische Projection repräsentirt (s. Fig. 1, 2, CR). Eine dritte Projection ist durch das Türck'sche Bündel als temporo-pedunculäre Bahn gegeben (Fig. 4, 5, 6, 7, TK). Schliesslich ist eine vierte Projection durch das sogenannte untere Längsbündel (fli in Fig. 4, 5, 6, $7,8)$ vertreten; hierüber ist Angesichts der sehr strittigen Auffassungen bezüglich der Bedeutung dieses Faserzuges eine kurze Erörterung nothwendig.

Nach der ältesten Ansicht wäre das untere Längsbündel ein mäcbtiges Associationssystem zwischen Hinterhaupt- und Schläfelappen. Diese Ansicht wurde in neuester Zeit von Flechsig und seinem Schüler v. Niess1-Mayendorf ${ }^{1}$ ) angegriffen, indem speciell letzterer auf Grund von Markentwickelungspräparaten sich folgend äussert. Es ist nach

1) v. Niessl-Mayendorf, Vom Fasciculus longit. inferior. Archiv für Psych. 1904. 
Flechsig vor Allem eine primäre Sehstrahlung zu unterscheiden, welche von den Autoren bisher irrthümlicherweise als Fasciculus longitudinalis inferior beschrieben wurde. Dieses Bündel stellt nach Flechsig und v. Niessl eine Projectionsfaserung des Hinterhauptlappens dar, welche im äusseren Kniehöcker und Sehhügel entspringt und ausschliesslich in der Fissura calcarina endigt, daher sei ihre Leitung eine centripetale. Ferner ist nach Flechsig noch eine secundäre Sehstrahlung zu unterscheiden, welche von der primären medialwärts liegt; sie erhält ihre Markhülle später. Thre Leitung soll grösstentheils eine centrifugale sein und entspricht der Sehstrahlung der Autoren. Sie entspringt aus der Fissura calcarina, der dritten Occipitalwindung, dem hintersten Abschnitt des Gyr. fusiformis und aus dem Hinterhaupt; ihre Endigung findet im Sehhügel und im vorderen Vierhügel statt. Im Wesentlichen bestätigen diese Angaben die Untersuchungen von M. Probst ${ }^{1}$; namentlich kounte dieser Autor die wichtige Thatsache nachweisen, dass in einem Falle von Erweichung des linken Hinterhauptlappens, wo die Erkrankung allein die drei Hinterhauptwindungen befiel, hingegen die mediale Fläche des Occipitallappens frei liess, mittels Marchi der ventrale Antheil des Stratum sagittale occipitale entartet war, und die degenerirten Züge liessen sich in den äusseren Knieböcker, zum Theile auch in das Pulvinar und in den lateralen Sehhügelkern hinein verfolgen. - Endlich Dejerin $\mathrm{e}^{2}$ ), übrigens ein Verfechter der partiellen Associationsnatur des unteren Längsbündels, beschreibt in diesem verlaufend ein ansehnliches Projectionsbündel, welches er "faisceau temporo-thalamique d'Arnold" nennt, und welches mit dem Türckschen Bündel zusammen das sublenticuläre Segment der inneren Kapsel ausmacht. Bezüglich des Arnold'schen Bündels giebt Dejerine folgende Schilderung. Es entspringt aus den Temporalwindungen, erhält ein wichtiges Fasercontingent aus dem Hinterhauptlappen und entartet partiell auf Läsionen des Gyr. lingualis und fusiformis. An schräghorizontalen, mit dem Sebtract parallel verlaufenden Schnitten erscheint nach Dejerine das Arnold'sche Bündel als ein parabolischer Faserzug, dessen Kuppe dem äusseren Theil des Mandelkerns entspricht, von hier ans zuräck- und aufwärts schlägt, um im Pulvinar und äusseren Kniehöcker zu endigen. Dieser Arnold'sche Tract entartet corticofugal.

Diese Angaben musste ich anführen, um die an meinen Präparaten

1) M. Probst, Zur Kenntniss des Sagittalmarkes und der Balkenfasern des Hinterhauptlappens. Jahrbücher f. Psychiatrie. 1901.

2) Dejerine, Centres nerveux. II. p. 37. 
geschilderten Verhältnisse fassbar zu machen. Der am meisten in's Auge springende Umstand ist so an den frontalen, besonders aber horizontalen Scbnitten der Mangel einer Sehstrahlung der Autoren (nach Flechsig's Terminologie, der secundären Sehstrahlung); die Componenten dieser Thatsache sind 1. das einestheils markfaserleere Pulvinar (S. Taf. IV, Fig. 5 und Taf. V, Fig. 4, 5R), 2. der Mangel eines von hier aus entspringenden und die äussere Wand des Unterhorns einsäumenden Bündels (Sagittalmark des Hinterhauptlappens). Ich möchte besonders darauf aufmerksam machen, dass diese äussere Ventrikelwand des Unterhorns weder ein äusseres, noch ein inneres Sagittalmark, also weder die Flechsig'sche primäre, noch secundäre Sebstrahlung aufweist; ebenso fehlt hier das Tapetum. Durch den Mangel eines Sagittalmarkes des Temporo-Occipitallappens erscheint das Türck'sche Bündel in besonders klarer Darstellung. Der soeben erwähnte Abgang der Temporo-Occipitalprojection macht sich jedoch nur bis zu den basalen Schnitten (in horizontaler Richtung) bemerkbar; an letzteren (S. Fig. 7, besonders 8 , Taf. V) ist bereits ein hakenförmig gekrümmtes, das Unterhorn parallel umkreisendes Bündel als der Fase. longit. infer. der Autoren sichtbar (S. Taf. IV, Fig. 8). Dieses Bündel verläuft in verschiedenen Niveaus; während der rückwärtige occipitale Theil desselben bereits in der Höhe des Sehhügels als schmächtiges Bündel erscheint (S. Taf. V, Fig. 4, fli) und als solches bis zu den basalen Horizontalschnitten an derselben Stelle, nämlich am hinteren Eck des Unterhorns verharrt, ohne an der äusseren Ventrikelwand nach vorn zu ziehen, taucht der an der äusseren Ventrikelwand verlaufende Theil des hinteren Längsbündels erst an den basalsten Horizontalschnitten als compacter Zug auf (S. Taf. IV, Fig. 8), zieht nach vorn in das sublenticuläre Segment der inneren Kapsel, biegt hier, bis an den äusseren Theil des Mandelkerns angelangt, aufwärts und rückwärts (S. Taf. IV. Fig. 7, Cisl) und geht in ein Markfeld über, welches dem äusseren Kniehöcker an dessen latralen Seite eng anliegt, resp. in diesen hineinströmt. (S. Taf. V, Fig. 6, lateral von ge; Taf. IV, Fig. 4, W und Fig. 5, W.) Dieser Verlauf dürfte das soeben geschilderte Bündel mit dem Dejerine'schen „faisceau temporo-thalamique d'Arnold" identificiren. Ob dieser Strang mit dem Sehact etwas zu thun hat, vermag ich positiv nicht entscheiden; sein Ursprung nach Dejerine, sowie seine corticofugale Natur widersprechen einer solchen Annahme entschieden. Möglich, dass diese Bahn im Sinne von v. Niessl-Mayendorf's das "motorische Glied der corticalen Reflexbahn" darstellt, somit für einen corticofugalen Tract anzusehen wäre. Hier wäre besonders noch auf Probst's experimentelle Arbeiten hinzuweisen, aus 
welchen hervorging, dass das temporo-occipitale Sagittalmark, das Stratum sagittale occipitale Probst's aus zweierlei Neuronen, aus thalamocorticalen und cortico-thalamischen Neuronen sich zusammensetzt. Für den vorliegenden Fall ist für sicher anzunehmen, dass in demselben die thalamocorticalen Neuronen, d. h. eine corticopetale, sensible Sehstrahlung grösstentheils fehlt, hingegen cortico-thalamische Neuronen anzutreffensind. Aus dieser Thatsache ist die Blindheit unseres Falles ohne weiteres zu verstehen, wenn schon der extracerebrale Abschnitt der Sehbahn, besonders der Sehnerv, die Kreuzung, der Sehtract, sowie der äussere Kniehöcker normale Structurverhältnisse, namentlich normalen Markfasergehalt darbieten.

Bezüglich der Commissurensysteme wäre kurz nur soviel zu erwähnen, dass dieselben alle vorhanden sind. So vor allem der Balken, an dem als abnorm seine Dünne auffällt, wie dies besonders an Frontalschnitten ersichtlich ist. Daselbst lässt sich leicht feststellen, dass die Fasern des Balkenkörpers nur zu den der Fissura pallii anliegenden Windungen gelangen, namentlich die erste Frontal- und vordere Centralwindıng beider Hemisphären verbinden; während Balkenfasern zu den, die laterale Hemisphärenfläche bildenden Windungen fehlen. Hierdurch ist auch ein gewisser Ausfall in den centralen Markmassen gegeben.

An Frontalschnitten fällt ferner die Thatsache auf, dass der Balken aus zwei Schichten besteht; aus einer oberen, welche stärkere Fasern besitzt, mit welchen eben die Verbindungen der, der Fissura pallii anliegenden Windungen bewerkstelligt wird; ferner aus einer unteren Schicht, weiche dünne Markfasern führend, charakteristischerweise den Ventrikel einsäumt und immer in das Strat. zonale des Schweifkerns übergeht (Fasc. nuclei caudati; Subst. grise sousépendymaire-Dejerine). (S. besonders Taf. I. Fig. 1,2, Taf. II. 2, 3. Fnc.) Die vordere und hintere Ausstrahlung des Balkens, wie dies an Horizontalschnitten nachzuweisen ist, lassen sich besonders in den mehr basalen Ebenen leicht auffinden; namentlich zeigt das Balkenlknie eine gute EntwickeIung (s. Taf. IV. Fig. $1 \mathrm{Cc}^{t}$ und Taf. V. Fig. 6. Ccg), Der Balkenwulst hingegen scheint nur in seinen basalen Ausstrahlungen den normalen Verhältnissen sich zu nähern, während in den höberen Ebenen sich eine nicht unerbebliche Reduction zeigt. - Die vordere Commissur bietet normales, wenn schon etwas geschwächtes Ausseben dar.

Schliesslich sei herrorgehoben, dass die Faserverhältnisse des Zwischen- und Mittelhirns normale sind. Besonders wäre daranf hinzuweisen, dass der Sehhügel, namentlich in seinem medialen Kern keinen Faserausfall zeigt, mit Ausnahme des Pulvinars, worüber oben schon Erwähnung gethan wurde. Auch ist der Pedunkulus normal mark- 
haltig und da wäre speciell auf die normal markreiche Pyramidenbahn hinzuweisen.

Nachdem wir nun die deskriptiven Einzelheiten des vorliegenden Falles von amaurotischer Idiotie kurz anführten, drängt sich nun die Frage auf: welcher Natur sind die geschilderten Abnormitäten? Um dieselbe zu beantworten, ist es rathsam die anatomischen Hauptzüge zu fixiren. Das Gehirn bot äusserlich keine bemerkenswerthen Anomalien in der Furchung und Windungsbildung dar; allein in der iuneren Structur fielen hauptsächlich zweierlei Abnormitäten auf. Ersstens fehlt das temporo-occipitale Sagittalmark in seiner Hauptmasse, so dass weder Flechsig's primäre, noch secundäre Sehstrahlung sichtbar ist. Zweitens macht sich ein nicht unbedeutender Mangel an den centralen Markmassen bemerkbar, und zwar lauptsächlich im frontalen und temporoparietalen Lappen; auf diese Weise entstehen guirlandenartige Windungen, welche allein durch fibrae propriae sich verbinden (s. besonders Taf. II. Fig. 2 von $\mathrm{F}_{1}$ zu $\mathrm{F}_{3}$; von $\mathrm{S}$ zu $O \mathrm{~m}$; ferner daselbst Fig. 3 von $\mathrm{F}_{1}$ zu $\mathrm{F}_{3}$, von $\mathrm{Tp}$ zu $\left.0 \mathrm{~m}\right)$. Tch denke nicht fehl zu gehen, wenn ich diesen zwei Hauptmängeln die zwei klinischen Ausfallserscheinungen zuschreibe; die Amaurose ist durcb die fehlende Sehstrahlung, die Idiotie durch den mangelhaften Associationsapparat bedingt. Bezüglich der Markfaserung der Hirnrinde kann ich soviel bemerken, dass dieselbe mit Normalpräparaten eines Erwachsenen (30 Jahre alt) verglichen, bezüglich der Structur sowie des Reichthums der Markfasern hinter letzterem nicht zurückbleiben. So ist z. B. in der exquisit-motorischen vorderen Centralwindung die supraradiäre Schicht durch die Gegenwart der zahllosen feinsten Fäserchen schon makroskopisch lichtblau; die Zonalschicht ist durch zahlreiche mittelstarke und stärkere Fasern gut vertreten; endlich die Radiärschicht wird durch stämmige Radii und durch einen stark entwickelten Interradiärfilz gebildet. Die Cuneusrinde weist eine dichte. Zonalschicht, einen gut entwickelten Baillarger'schen Streifen, eine zarte, doch dinstincte Supraradiärschicht und tannenartig dicht nebeneinandergereihte Markstrahlen auf. Die tiefe Temporalwindung zeigt eine kräftige, makroskopisch sehr ins Auge fallende Zonalschicht, in der aus tangentiell verlaufenden mittelfeinen Fasern gebildeten Supraradiärschicht noch einen gut ausgeprägten Kaes'schen Streifen; an der oberen Grenze der Radiärschicht macht sich noch ein Baillargerscher Streifen bemerkbar. Colossal starke, förmlich gigantische Zonalschicht ist am Temporalpol, wamentlich an dessen der Insel zugekehrten, also Innenfläche zu sehen. Die Insel hat eine breite, lockere Zonalschicht, schwächere Markstrahlen, eine mehr faserarme Supraradiär- 
schicht, an deren unteren Grenze 1-2 gut angedeutete Baillargersche Streifen auffallen. Die basalen Frontalwindungen weisen eine kräftige Zonalschicht und an der Grenze zwischen Radiär- und Supraradiärschicht einen kräftigen Baillarger auf. - Durch diese Beispiele denke ich genügend nachgewiesen zu haben, dass Bildungsfehler in der Markstructur keine Rolle spielen; an einer einzigen Stelle, namentlich an Fig. 2, Taf. II gekennzeichnet durch zwei Sterne, erscheint die tiefe Rinde der hinteren Centralwindung in ihrer Faserstructur wie zerzanst, ein Verhalten, welches an der Grosshirnrinde bei Idiotie geradezu normal zu nennen ist.

Aus obigen Angaben ist ersichtlich, dass im Gehirn des vorliegenden Falles von amaurotisch-spastischer Idiotie Entwickelungshemmungen die ausschlaggebende Rolle spielen, denn nur so sind die oben angeführten Mängel: symmetrisch-defecte centrale Marksubstanz und fehlende Sehstrahlung verständlich.

Für die Entwickelungshemmung spricht vor Allem die scharf hervorzuhebende Thatsache, dass das Centralnervensystem im Uebrigen normal gebildet ist. Heinrich Vogt, der die Ziele und Wege der teratologischen Hirnforschungsmethode vorzüglich entwickelte, ${ }^{1}$ ) weist auf den zuerst von ihm formulirten Umstand hin, dass das Wesen der böher differenzirten Missbildung in der Fixation eines Theils der Anlage gegenüber den übrigen liegt; der fixirte Theil wird in seiner Weiterentwicklung gebemmt, während die übrigen Theile den gewohnten Gang der Entwickelung bis zur Vollentwickelung durchmachen. Dieser Autor zerlegt den Process der Entwickelung erstens in eine formative Phase, in welcher die Anlage eines Organs vor sich geht, wie die Höhlenbildung, Abschnürung, Knickung; hier „herrschen die Vorgänge der Gestaltung der äusseren Form" (1. c.). Nach dem dritten Embryonalmonat wird diese formative Phase durch die von H. Vogt Organogenesis genannte Phase abgelöst. „In dieser treten innerhalb der durch die formative Phase abgegrenzten Zellmasse Umlagerungen auf. Das Bild ist, da die Entwickelung eines Organs in allen Theilen ein synchroner Process ist, ein sehr gleichartiges; greifbare Abgrenzungen und Unterscheidungsmerkmale fehlen, weder Betrachtung noch histologische Gesichtspunkte, noch Reconstruction kann hier Einblick gewähren. . . . . Das Problem besteht darin, aus der Gleichartigkeit der Zellmassen einzelne Theile zu isoliren. Diese Forderung erfüllen die Missbildungen (für die organogenetische Phase die höher differenzirten Missbildungen)"

1) H. Vogt, Ueber Ziele und Wege der teratologischen Hirnforschungsmethode. Monatsschr. f. Psych. u. Neurol. Bd. XVII. H. 4. 
(l. c. S. 422, 423). - Wir können daher im Sinne der H. Vogt'schen Ausführungen den vorliegenden Idiotiefall für eine hoch differenzirte Missbildung betrachten; dieselbe kam dadurch zu Stande, dass die zentralen Markmassen des Frontal- sowie des Parietotemporallappens, ferner ein gewisser Theil des Projectionssystems, namentlich die Sehstrahlungen, in ihrer Entwickelung ganz gehemmt wurden, oder präciser ausgedrückt, aus dem Entwickelungsgang (aus Vogt's organogenetischer Phase) einfach ausblieben, welcher Umstand die synchrone Entwickelung benachbarter Theile gar nicht oder unwesentlich beeinflussend, ein Gehirn entstand, welches im Uebrigen in seiner Architektonik normal erscheint. Es handelt sich daher für den vorliegenden Fall um eine reine Bildungshemmung; welcher Umstand die Hemmung veranlasste, oder mit $H$. Vogt's Worten, worin das „primäre pathologische Moment" besteht, entzieht sich meiner Erkenntniss. Nach H. Vogt's zutreffender Bemerkung ist das missbildete Organ ein krankes Organ, welches zu secundären Erkrankungen, Blutungen aus den dünnwandigen und oft selbst schon krankhaft veränderten Gefässen neigt. Somit ist für Missbildungen die Möglichkeit von Erkrankungen zweiter Ordnung gegeben; diese Erscheinung nennt H. Vogt das ,secundäre pathologische Moment." Für meinen Fall bedarf es keines besonderen Nachweises, dass in demselben das secundäre pathologische Moment keine Rolle spielt; zwar wurden bei der Herstellung der Schnitte die Zellfärbungen leider unterlassen, doch ist so viel festzustellen, dass gröbere histopathologische Veränderungen ganz fehlen. Ein Beispiel für das secundäre pathologische Moment dürfte der Fall M. Friedmann's geben ${ }^{1}$ ); hier bestanden von früher Jugend auf spastische Paraplegie und Idiotie mit geringen Sprachrudimenten; in den 20 er Jahren traten Convulsionen epileptischen Charakters auf; endlich im 30. Jahre wurde durch Lungenentzündung bei hochgradigem Marasmus der Tod herbeigeführt. Mikro- und makroskopisch fanden sich folgende Veränderungen vor: Leichtere frische und alte Pachymeningitis, namentlich aber immense Atrophie des Hemisphärenmarks; überall äusserst stark verbreitete hyaline Entartung der kleinen Blutgefässe, eine Unzahl kleiner, fibrillärer, von Nervensubstanz freier Degenerationsherde, woraus auf- und absteigende Entartungen (u. A. jene der Pyramide) resultirten. Auf diese Weise erlitt das ganze Hemisphärenmark eine Reduction auf ein Fünftel der Norm; dieselbe geschah hauptsächlich auf Kosten der Projectionsfasern,

1) M. Friedmann, Ueber einen Fall von mit Idiotie verbundener spastischer Paraplegie im Kindesalter mit Sectionsbefund. Dtsch. Zeitschr. f. Nervenheilkunde. Bd. III. 1892. 
während die Fibrae propriae verschont blieben, so dass die Windungen meist nur mit schmaler, stielartiger Basis dem Marke aufsitzen und von den Basalganglien durch eine sehr schmale Stabkranzregion getrennt sind. Auf diese Weise kamen Windungsbilder zu Stande, welche den meinigen auf' den ersten Blick ungemein ähnlich sind; doch trennt beide Bilder ein fundamentaler Umstand: Friedmann's stielartig dem Marke aufsitzende Windungen entstanden in Folge der aus der disseminirten hyalinen Gefässerkrankung resultirenden Markatrophie (secundäres pathologisches Moment), während die ebenfalls stielartig dem Marke angefügten Windungen meines Falles ihre Genese der Markentwickelungshemmung (primäres pathologisches Moment) verdanken. Im Sinne dieser Hemmung sind auch jene zumeist gefässführende Spaltbildungen zu deuten, welche in den einzelnen Lappen erscheinen (s. Taf. IV, Spaltungen der $\mathrm{Ca}, \mathrm{T}_{1}$; Taf. V, Fig. 1, zwischen Pre und Os; Fig. 2, 3, ep im Hinterhauptslappen usw.); sicherlich sind das Ueberbleibsel der embryonalen Höhlen, welche selbst in dieser rudimentären Form durch das normal entwickelte tiefe Mark verwischt werdeu, hier aber eben mangels centraler Markmassen als Spaltbildungen persistiren.

Nachdem die Blindheit und Idiotie meines Falles durch die nachgewiesenen Entwickelungshemmungen vollkommen erklärt werden, erfordern noch die spastisch-paralytischen sowie die convulsiven Erscheinungen ein histopatbologisches Substrat. Und da sei vorweg bemerkt, dass eine fassbare Unterlage für letztere Symptome nicht gegeben werden kann. Ich möchte hierbei vor Allem die spastische Lähmung, besser gesagt die hochgradige Contractur der unteren sowie oberen Extremitäten einer Erörterung unterziehen. Oben sahen wir, dass die Pyramidenbahn anatomisch vollkommen intact ist und doch sehen wir den höchsten Grad von Hypertonie in unserem Falle; es ist dies ein Vorkommen, welches beweist, dass zur Entwickelung spastischer Zustände die Affection der Willensbahn nicht immer nothwendig ist. Denn wie Blocq in seiner die Contracturen behandelnden Arbeit sehr richtig hervorhebt, giebt es einmal Rigiditäten ohne Erkrankung der Pyramide, zweitens muss die Pyramidenaffection nicht unbedingt durch Contractur gefolgt sein. Mein Fall ist ein Beispiel für spastische Zustände ohne Pyramidenerkrankung; zur Erklärung dieses Verhaltens denke ich am richtigsten die Adamkiewicz-Anton'sche Auffassung bezüglich des Wesens der Contractur heranzuziehen. Im Sinne dieser Lehre bedeutet der spastische Zustand eine fehlende cerebrale Innervation bei überwiegender spinaler Innervation, indem unter normalen Verhältnissen der Muskeltonus durch die Pyramide und durch die sensiblen Rückenmarksbahnen antagonistisch beeinflusst wird. Eine jedwelche cerebrale 
Läsion kann zur Contractur ohne Pyramidenläsion führen, denn die cerebrale Dämpfung, das Resultat der gesammten Hemisphärenthätigkeit, wird in diesem Falle vermindert. Die Willensbahn (deren einer, und zwar vornehmster Theil die Pyramide ist) ist nur das Instrument, welches diese Dämpfung zur Musculatur leitet. Auf diese Weise ohne mich in das Wesen der Contractur zu vertiefen - stelle ich mir in meinem Falle die Contractur entstanden vor; dieselbe ist also ein patho-physiologisches Product der mangelnden cerebralen Innervation, bedingt durch das defecte Grosshirn.

Während trotz fehlender Pyramidenaffection die Contractur verständlich ist, mangelt uns für die epileptiformen Erscheinungen unseres Falles eine naheliegende Erklärung. Anatomische Veränderungen seitens der Centralwindungen, des Ammonshorns fehlen; es bliebe nur eine dynamische Interpretation übrig, welche uns aber allzusehr in das Gebiet der Hypothese führen dürfte. Empirisch ist aber die Thatsache von convulsiven Erscheinungen bei Idiotie nicht im mindesten überraschend.

Die mangelnde cerebrale Thätigkeit des vorliegenden Falles giebt sich endlich in den hochgradigen trophischen Störungen des Muskel- und Knochengewebes kund; besonders die Huskeln blieben in ibrer Entwickelung hochgradig zurück (s. Krankengeschichte). Wie sehr diese Abnormitäten des Muskelvolums einfache Entwickelungshemmungen und keine degenerativen Erscheinungen sind, beweist das elektrische Verhalten der Extremitätenmuskulatur, welches als normal zu bezeichnen ist; keine Spur von einer Entartungsreaction.

Um das Wesentliche des geschilderten Falles herauszugreifen, wiederhole ich nur so viel, dass es sich um eine amaurotisch-spastische Idiotie handelte, welche bei fortschreitendem Marasmus durch eine Pneumonie beendet wurde. Der Fall besitzt jene Charakterzüge, welche H. Vogt (1. c.) für seinen einheitlichen Typus von familiärer amaurotischer Idiotie als kennzeichnend erachtet. Es war die spastische Paraparese der vier Extremitäten, die Diplegie der Autoren, ferner die hochgradige Abnahme der Sehkraft, dann der psychische Defect und endlich der fortschreitende Marasmus vorhanden. Ein einziger Zug konnte mangels anamnestischer Daten nicht festgestellt werden, die Familiarität des Leidens. Ich möchte aber an dieser Stelle bemerken, dass trotz fehlender Anamnese dieser letzte Zug mit sehr grosser Wahrscheinlichkeit, wenn nicht mit Sicherheit für den vorliegenden Fall auszuschliessen war. Diese Vermuthung schöpfe ich aus der pathologischen Art des Falles; Entwickelungshemmungen dieser Form tragen einen bezüglich der Entstehung derartig individuellen Stempel an sich, dass sie immer nur ein "Fall" bleiben; es fehlt ihnen etwas Generelles, 
wodurch die Wiederholung in einem engen Kreise, in einer Familie, wenn schon nicht absolut ausgeschlossen, jedoch höchst unwahrscheinlich ist. Also nicht so, wie bei der Sachs'schen oder Spielmayerschen Form der Idiotie, wo es sich um eine generelle abnorm schwache Veranlagung der Nervenzellen des gesammten Centralnervensystems handelt; hierüber sofort noch mehr.

Der vorliegende Fall ist somit als eine spastisch-amaurotische Idiotie ohne Familiarität zu charakterisiren, differirt daher rom H. Vogt'schen Typus eben durch den Mangel an Familiarität. Diese klinische Abweichung giebt sich aber auch in anatomischer Beziehung kund, wie dies sich bei einem Vergleich der beiden Formen sofort herausstellt. Den Sachs'schen Fällen geht jede grob-makroskopische Veränderung, in erster Linie aber Hemmungsbildungen von Bedeutung: vollkommen $a b$, vielmehr sind dieselben nur eytopathologisch charakterisirt, indem ein ganz normal entwickeltes Centralorgan im Verlauf des 1. oder 2. Lebensjahres in Folge abnorm frühzeitigèr Abnützung des nicht lebensfähigen Zellprotoplasmas, eine auf das gesammte Centralgrau sich erstreckende Degeneration erfährt. Allein die Nervenzellen sind jene Elemente, welche im Centralorgan der Sachsschen Kranken abnorm schwach und für die weitere Entwickelung unfähig veranlagt sind, welcher Thatsache B. Sachs1) mit folgender Definition gerecht wird: "We may simply argue that children who are afflicted with Amaurotic Family Idiocy have a very limited capacity for normal development; that their central nervous system is not equal to the functions that they are expected to perform for more than the first three or four months of life, and from that time on a rapid deterioration of all functions is established." - Im Gegensatz hierzu stellt mein vorliegender Fall von amaurotischer Idiotie des Jugendalters etwas ganz Verschiedenes dar; bier handelt es sich nicht um cellularpathologische, sondern um grob-anatomische Veränderungen, welch' letztere nicht als eine progressive Entartung des gesammten Centralorgans erscheinen, sondern in der Form einer aus der organogenetischen Phase stammenden hochdifferencirten Missbildung des Vorderhirns auftreten. Die Art dieser Nissbildung, die Localisation der "Fixation",

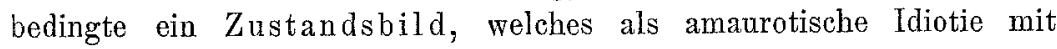
spastischer Paraparese und convulsiven Erscheinungen cbarakterisirt werden kann; denn dadurch, dass die Hemmung im Entwickelungsgange des Vorderhirns gerade die Sehstrahlung resp. die centralen,

1) B. Sachs, A treatise on the nervous diseases of ehildren. Second edition. New-York. 1905. p. 468. 
Associationssysteme betraf, entstand die Amaurose resp. die Idiotie. Somit ist die klinische Aehnlichkeit dieses Falles mit den Sachs'schen Fällen nur eine äusserliche und hat mit dem inneren Wesen der letzteren gar nichts Gemeinsames. Anatomisch lässt sich die Differenz derartig formuliren, dass, während die Sachs'sche Form der amaurotischen Idiotie eine cytopathologisch charakterisirte Form ist, erscheint die im vorliegenden Fall beschriebene ldiotie als eine teratologisch begründete Form.

Bevor ich schliesse, sei es mir gestattet, nochmals auf $\mathrm{H}$. Vogt's gemeinsamen Typus zurückzugreifen, in welchem, wie bereits erwähnt, zwei Formen zu unterscheiden wären, nämlich die infantile oder Sachssche, und die juvenile Form, wohin H. Vogt die in neuester Zeit von W. Spielmayer beschriebene Form auch rechnet. Ich möchte diese zwei Formen einer klinisch-anatomischen Parallele unterziehen, und da H. Vogt's anatomische Untersuchungen bezüglich der juvenilen Form noch ausstehen, so denke ich am richtigsten die vorliegenden klinischhistologischen Daten Spielmayer's heranzuziehen. Ich kaun dies um so berechtigter thun, da Herr College Spielmayer die besondere Freundlichkeit hatte, mir einige gelungene Präparate aus zwei Fällen seiner Form gütigst einzusenden und ich somit in der Lage war, auf Grund von eigener Vergleichung dem bistopatbologischen Substrat der infantilen oder Sachs'schen Form jenes der juvenilen, auch Spielmayerschen Form gegenüberzustellen.

Bezüglich des klinischen Verhaltens möchte ich nebst der unleugbaren allgemeinen Uebereinstimmung, welche in der spastisch-familiären Amaurose gegeben ist, noch auf einen in nosographischer Beziehung nicht unwesentlichen und abweichenden Umstand aufmerksam machen, worunter ich den Augenspiegelbefund verstehe. In den von Spielmayer beobachteten Fällen handelte es sicb um vier Kinder einer Familie, die bis zum Ausbruch der Krankheit körperlich und geistig normal erschienen. Mit dem sechsten Jahre begann die psychische Veränderung, die Idiotie und ziemlich gleichzeitig verlief eine progrediente Atrophie der Netzhaut vom Typus der Retinitis pigmentosa. Bezüglich des Krankheitsverlaufs berichtet Spielmayer, dass die Idiotie sich rasch entwickelte; irgendwelche Lähmungserscheinungen, die auf eine infantile Paralyse hinwiesen, wurden nie beobaehtet. Von vier Kindern kamen drei so ziemlich in demselben Lebensalter, in den ersten Pubertätsjahren zur Section. Bezüglicb der Aetiologie misst Spielmayer der Syphilis eine gewisse Bedeutung bei, da der Vater vor der Zeugung der kranken Kinder sich luetisch inficirte; sein erstes Kind, welches vor diesem fatalen Ereigniss gezeugt wurde, blieb gesund. Spie]mayer bezeichnet 
dieses Krankheitsbild nach seinen klinischen Grundzügen als eine familiäre amaurotische Idiotie, thut dies jedoch mit folgender Bemerkung: "Damit soll aber nichts über die Zugehörigkeit dieses Processes zu der schlechthin so benannten familiärell, amaurotischen Idiotie, der Sachsschen Krankheit, ausgesagt sein." Ferner: „Ich habe schon an anderer Stelle dargelegt, dass meine Fälle mit dieser Krankheit nichts weiter als die rein äusserliche Eigenthümlichkeit gemeinsam haben, dass sie beide familiär auftreten und dass sie zu frühzeitiger Verblödung und zur Erblindung führen. Sonst sind sie klinisch durchaus verschieden."1)

Wir sehen also, dass Spielmayer gegen eine Wesens-Identificirung der infantilen und juvenilen Idiotie Protest einlegt. Ich meinerseits möchte nur auf den Umstand hinweisen, dass ich den Naculabefund der Sachs'schen Idiotie für ein wichtiges Phänomen betrachte, dessen Gegenwart einen Idiotiefall nosographisch ungemein scharf charakterisirt. In unseren sieben, mit Herm Primar Dr. Julius Grosz gemeinsam beobachteten Fällen des Brody-Kinderhospitals in Budapest, constatirte Herr Docent Dr. H. Mohr, Primar der Augenkrankenabtheilung daselbst, in allen Fällen die charakteristische Fundusveränderung, welche bekanntlich darin besteht, dass in beiden Augen an der Stelle des gelben Fleckes ein ca. $1 \frac{1}{2}$ Papillen breiter, weisser Fleck, im Centrum mit kirschrothem Tupf, entsteht. Von besonderer. Wichtigkeit ist der Umstand, dass in allen unseren Fällen der Maculabefund als solcher usque ad finem persistirte, nur in einigen gesellte sich noch eine Opticusatrophie hinzu. Letztere wurde ohne Macularveränderung nie erhoben, daher sind meines Erachtens die amaurotisch-familiären Idiotien mit einfacher Opticusatrophie nosographisch nicht mit der Sacbs'schen Idiotie in einen Topf zu werfen. Die eingangs erwähnten Fälle von Mülberger, Heveroch und Koller, amaurotische Idiotien ohne Maculabefund, jedoch mit atrophischen Papillen, schliesse ich daher aus dem Rahmen der Sachs'schen Idiotie ganz entschieden aus; mit anderen Worten: eine familiär-amaurotische Idiotie gehört nur dann zur Sachs'schen Form, wenn sie die charakteristische Fundusveränderung aufweist, daher betrachte ich letztere nosographisch als ein specifisches Charakteristikum. Wenn daher H. Vogt der Meinung ist, dass "nevere Beobachtungen den Maculabefund nicht als eine conditio sine qua non für die Diagnose kennen gelernt haben" (1. c. S. 313), so muss ich dieser Auffassung entschieden widersprechen. Wenn ich nun das Facit aus der klinischen Parallele ziehe, so lässt

1) W. Spielmayer, Ueber eine besondere Form von familiärer amaurotischer Idiotie. Neurol. Centralbl. 1906. No. 2. 
sich folgendes sagen: Die Hauptzüge, wie Blindheit, Lähmung und Verblödung, sind der infantilen wie juvenilen Form der familiär-amaurotischen Idiotie gemeinsam, duch grenzt sich die Sachs'sche Form von allen übrigen Formen der familiär-amaurotischen Idiotien durch den Naculabefund scharf ab. Wie sehr der Fundus oculi für einzelne Formen sich charakteristisch verhalten kann, zeigt die Spielmayersche juvenile Form, die eine Netzhautveränderung aufweist, welche den von H. Vogt beschriebenen gleichfalls juvenilen Fällen nicht eigen ist. Allerdings macht Spielmayer selbst die Bemerlzung, es wäre durchaus denkbar, dass die Netzhautatrophie lediglich die Bedeutung eines Begleitsymptomes hätte und beendet seinen Aufsatz wie folgt: „Bei den Versuchen einer Lösung dieser Frage (über die Bedeutung der retinalen Veränderung) wird die pathologische Anatomie den Ausschlag geben müssen; denn das anatomische Substrat ist in dieser Aufgabe der gegebene Factor, und die Frage lautet deshalb: "Welches sind die klinischen Grundzüge dieses Processes, dessen an a tomische Sonderstellung diese Untersuchung ergab?" Spielmayer bezieht sich mit letzteren Worten auf die histologische Untersuchung zweier seiner Fälle und hält das Ergebniss, das histologische Gesammtbild, für dermassen eigenartig, dass eben auf Grund des anatomischen Befundes seine Form von familiär-amaurotischer Idiotie als selbstständige Krankheitsform aus der Reihe der idiotischen Zustände abzusondern wäre. Somit bin ich an die histologische Parallele der infantilen und juvenilen Formen der familiär-amaurotischen Idiotien, an den Schwerpunlkt jener Frage angelangt, ob H. Vogt's Zusammenfassung zu Recht bestehe.

Den histologischen Befund der infantilen oder Sachs'schen Form erwähnte ich auf Grund eigener Untersuchungen bereits. Der grundlegende Prozess ist eine hochgradige Schwellung so des Leibes wie der Fortsätze der Nervenzellen des gesammten Centralnervensystems, welche mit Nissl's Färbung, wie dies zuerst B. Sachs und ich nachwiesen, als eine intensive Chromolyse sich kundgiebt, wodurch dann jenes Gerüst, welches durch die Nisslkörper incrustirt wird, Cajal's Spongioplasma deutlich zum Vorschein gelangt. (Siehe meine Schilderung im Journal f. Psychol. u. Neurol. 1905. Bd. VI.) Interessante Bilder liessen sich mit Bielschowsky's Fibrillenfärbung gewinnen, denn obschon die Nisslpräparate Schwelluugen des Zellkörpers ergaben, sowie auf Formveränderung der primären Protoplasmafortsätze hinwiesen, so demonstrirte erst die Silbermethode mit Klarheit das nähere Verhalten der Dendriten, welches ich speciell mit Rücksicht auf die Fibrillenbilder der Spielmayer'schen juvenilen Form von Belang erachte. Schon in der soeben erwähnten, ferner in einer demnächst 
a. a. O. erscheinenden Arbeit beschreibe ich des Näheren die Einzelheiten dieser Schwellung, welche in aller Kürze gefasst derart sich gestalten, dass in der ersten Phase des Processes besonders das Donaggio'sche endocellulare Netz durch die Aufblähung der Netzmaschen deutlich sichtbar wird, nebstbei ist eine grosse Hypertrophie der Netzknotenpunkte auffallend. In der zweiten und späteren Phase zerbröckelt sich das Innennetz der Nervenzellen, so dass es wie aus zahlreichen Körnern zusammengesetzt erscheint; später verwandelt sich dies desintegrirte Innennetz in eine dichte, diffuse Staubmasse, welche in der Endphase des Processes zur successiven Aufhellung, schliesslich zum totalen Schwund gelangt, wobei aber die Aussen- oder Golginetze noch erhalten bleiben. In der geschilderten Weise verläuft der Entartungsprocess so im Zellleib wie in den Dendriten; die Schwellung bedingt, wie ich dies bereits erwähnte, ganz colossale Aufblähungen (meine "cystische Degeneration"), welche in besonders instructiver Weise an den Fortsätzen zur Darstellung gelangen. Ein contrastirender Zug ist in diesem Process das Verhalten des Axencylinders, denn dieser nimmt an der Schwellung nicht theil, verhält sich daher als Corpus separatum. Der Process ist der denkbar diffuseste, denn man findet keinen verschonten Abschnitt der centralen grauen Massen, vom Rindengrau bis zum Conusgrau binab.

Ich gehe nun za Spielmayer's Befunden über; sehen wir, worin das anatomische Substrat der juvenilen Form der familiär-amaurotischen Idiotie besteht. Vorweg sei bemerkt, dass Spielmayer in seinen Fällen einen makroskopisch negativen Befund erhob, gleich wie ich dies für die Sachs'schen Fälle, im Gegensatz zur verbreiteten Voraussetzung der Autoren, als erster mit besonderer Betonung hervorhebe. Mikroskopisch fand Spielmayer "eine eigenartige, ganz allgemein über die Centralorgane ausgedehnte Ganglienzellenerkrankung, die durch eine totale oder partielle Schwellung oder Aufblähung des Zellleibes in Folge der Einlagerung einer körnigen Substanz charakterisirt ist". Diese "abnorme Zellsubstanz" ist manchmal pigmenthaltig und ist am ausgesprochensten in der Grosshirnrinde, "dort combinirt sie sich auch mit verschiedenen der bekannteren Erkrankungsformen (chronische, sklerotische Zellerkrankung, Zellschattenbildung, Neuronophagie, Incrustationen der Golginetze u. s. w." Spielmayer findet für auffallend das Intactbleiben der Projectionsfaserung und besonders das nahezu normale Aussehen des Markscheidenund Axencylinderbildes.

Wie ich bereits oben erwähnte, liess mir die Freundlichkeit Spielmayer's mehrere Präparate seiner Fälle zulommen und so möchte ich. 
an dieser Stelle nur in aller Kürze erwähnen, dass ich an seinen Fibrillenpräparaten (gleichfalls mit Bielschowsky's Färbung!) die von ihm beschriebene Aufblähung des Zellkörpers, sowie an Nisslpräparaten seine "Einlagerung einer körnigen Substanz" gleichfalls auffand, aber bezüglich der Interpretation letzterer Substanz von ihm abweiche. Bei einer Analyse mit Zeiss Apochromat $2 \mathrm{~mm}$ und Compensationsocular 18 lässt sich der Nachweis sehr leicht erbringen, dass an Stelle Spielmayer's "körniger Substanz", welche er seiner Schilderung nach als ein abnormes Product sui generis auffasst, das durch die Schwellung deutlicher gemachte Innennetz (Donaggio's endocelluläres Netz) sich befindet, daher die "wabig angeordneten Stippchen", welche Spielmayer selbst aufgefallen sind. Dieses Innennetz zeigte sich in besonders instructiver Form an den Rückenmarkszellen der Spielmayerschen Präparate; daselbst, aber deutlich auch an den Grosshirnzellen, war ausserdem das Aussennetz erkennbar, und zwar hier überwiegend als fasciculo-fibrillär gebaute Substanz. Diese zwei Substanzen sieht man an Fig. 2 der Spielmayer'schen Arbeit (1. c.), an einer Beetzschen Pyramide klar wiedergegeben; man sieht dort einestheils die deutlich reticulirte, aus sehr feinen Fäden gesponnene Innensubstanz (endocelluläres Netz), welcber die fasciculo-fibrilläre Aussensubstanz (Aussenuetz) aufliegt. Von einer körnigen Substanz verrathen Spielmayer's Fibrillenpräparate nichts; diese bekommt man erst an Niss1präparaten zu Gesicht. Was Spielmayer an seinem Nisslbilde (Fig. 1, l. c.) als körnige Substanz mit wabig angeordneten Stippchen wiedergiebt, dürfte meines Erachtens nur Pigment sein, welches an dieser Stelle die netzartig vertheilte und verfeinerte Nisslsubstanz durchdringt. Vor 10 Jahren (1896) batte ich auf dieses charakteristische Verhalten der Nisslkörner bereits hingewiesen ${ }^{1}$ ) und im Jahre 1898 gelegentlich der Beschreibung der Nervenzellenveränderungen des Vorderhorns bei Tabes ${ }^{2}$ ) diese Erscheinung wiederholt geschildert. An letzterer Stelle äusserte ich mich folgend: „Die chromatische Substanz, wie dies meine Beobachtungen ergaben (s. Revue Neurologique. 1906. 1. c.) erleidet in der Nähe des Pigments und besouders in demselben eine auffallende Grössenveränderung, indem die chromatischen Granula gegen das Pigment zu, gleichwie in der Nähe des Axons, entschieden kleiner werden, während sie im Pigmente selbst nur als kleinste, rundliche, auch sternförmige Körnchen erscheinen. An mehreren Präparaten hatte es den Anschein, als wïrden diese klein-

1) Sur l'origine de l'amyotrophie tabétique. Revae Neurolog. 1896.

2) Monatsschr. f. Psychiatrie u. Neurolog. 1898. Heft 1. 
sten chromatischen Körnchen durch allerfeinste Füden zu einem Jetze verbunden, in dessen Maschen die sternförmigen Anschwellungen der chromatischen Substanz sich befinden" (1. c. S. 72). Ich halte num die in Fig. 1 Spielmayer's angedeutete, kugelige, scharf umschriebene, von einem feinen Netzwerk durchsponnene Masse entschieden für Pigment; hierauf weist übrigens Spielmayer selbst hin, da er die „abnorme Zellsubstanz" "hier und da" pigmenthaltig schildert. Da dieselbe aber auch die Scharlachreaction giebt, so könnte an eine Umwandlung des Pigments in eine fettige Substanz gedacht werden, obschon ich Spielmayer's brieflicher Mittheilung entnehme, dass die Scharlachreaction nur schwach ist und er eine deutliche Sudanreaction nicht erhielt. Somit erscheint die Fettnatur der körnig-pigmentösen Substanz fragwürdig. Im Gegensatz hierzu hebt Sträussler'1) hervor, dass die an Stellen der Schwellungen sichtbaren Granulirungen, welche ich als Fibrillendetritus auffasse, mit Rücksicht auf ihre Osmiumreaction, als Fettpigment zu betrachten sei. Die Granulirung ist nach ihm die erste Aeusserung der Zellerkrankung, welche Veränderung er als die fettig-pigmentöse Entartung der Zellen und protoplasmatischen Fortsätze bezeichnet. Gegen meiner soeben erwähnten Auffassung setzt er die Thatsache entgegen, dass die Körner bereits zu einer Zeit auftreten, wo ein Zerfall von Fibrillen sicher noch nicht stattgefunden hat. Hierauf bab ich nur soviel zu bemerken, dass der Detritus faktisch nicht aus dem reticnlo-fibrillären Aussennetz entsteht, welches ich eben für sehr wiederstandsfähig fand, sondern aus dem Zerfall des Innenuetzes, dessen Gegenwart und Bedentung Sträussler zu entgehen schien wenigstens die Existenz und Rolle desselben berührt er in seiner Arbeit nirgends. Die Mlöglichkeit, dass der Innennetzdetritus im Verlaufe der Zellentartung schliesslich eine fettartige Vetamorphose erleiden könne, will ich keineswegs in Abrede stellen, betonen möchte ich jedoch, dass es mir an meinen Marchi-Präparaten aus zwei Sachs'schen Fällen eine Schwärzung dieser Granulirung nicht gelang.

Um mich klar zu fassen, erlaube ich mir kurz Folgendes hervorzuheben. In den Nervenzellen, welche einer Schwellung unterliegen, entsteht im Verlaufe der Entartung ein doppelter Zerfallsprocess. In erster Linie zerbröckelt sich die ansonsten auch locker aggregirte Niss 1substanz, es entwickelt sich daher ein auf Rechnung der chromatischen Körner zu setzende mehr oder minder feine Staubsubstanz; in diesem

1) E. Sträussler, Ueber eigenartige Veränderungen der Ganglienzellen und ihror Fortsätze im Centralnervensystem eines Falles von congenitaler Kleinhirnatrophie. Neurol. Centralbl. 1906. No. 5. 
Stadium hat das Innennetz nur eine mässige Blähung erfahren, wäbrend das reticulofibrilläre Aussennetz noch ganz intakt dasteht. Spielmayer's Präparate entsprechen diesem Stadium der Zelldegeneration, folglich halte ich dafür, dass seine körnige Masse, wo sie in gelben Körnern erscheint, nichts anderes als Pigment ist, wo sie eine schwache Thioninfärbung zeigt, eigentlich zerfallenes Tigroid ist. In einem späteren und vorgeschrittenen Stadium leidet nun das Innennetz, in einer Weise, wie ich das schon oben schilderte; kurz, dieses zerfällt in Körner, wobei das Aussennetz, die vulgär Fibrillen genannte Substanz noch nicht gelitten hat. Daher bekommt man Bilder wie Sträussler sie schildert und wie auch ich dies für die Sachs'schen Fälle beschrieb: die sog. fibrilläre Substanz umgiebt korbartig oder hülsenartig die Zerfallmasse, i. e. das decomponirte Innennetz.

Aus dem soeben geschilderten Hergang ist es ersichtlich, dass die Nissl-Köruer bereits bei incipientester Degeneration sich krankhaft verändern; erst später folgt das Innennetz, zuletzt das fibrilläre Aussennetz. Auf dieses vom cytopathologischen Standpunkt hochinteressante Verbalten wies ich bereits a. a. 0 . mit besonderem Nachdruck hin. Hieraus ist es nicht! schwer zu begreifen, warum tigrolytische Nervenzellen noch functionstüchtig sind.

Vergleiche ich nun die Nervenzellenveränderungen der Spielmayer'schen Fälle mit meinen Sachs'schen Fällen, so muss ich im Allgemeinen die essentielle Uebereinstimmung hervorheben; bier wie dort dominirt ein Schwellungsprocess der Nervenzellen, welcher sich vor Allem durch die Auftreibung der Maschenlücken des intracellulären Netzwerkes kundgiebt. Die histologische Differenz zwischen den beiden Formen ist nur eine graduelle, keineswegs eine essentielle; in Spielmayer's juveniler Form gelangt es nicht zu solehen excessiven Formen der Schwellung wie bei der Sachs'schen infantilen Form, namentlich aber fehlt dort vollkommen die Aufblähng der Dendriten und ausserdem sah ich bei ersteren auch nicht jene hochgradige Entartung des Innennetzes, welches, zur Entwickelung einer Staubmasse somit zur Vernichtung des Netzes führt. Auch ist die consecutive Gliawucherung bei Sachs'scher Idiotie viel bedeutender wie bei der Spielmayerschen Form. In Spielmayer's Fällen spielt sich also derselbe Process ab, wie in den Sachs'schen Fällen, nur erreicht derselbe in ersteren keineswegs jene Intensitat wie in letzteren. Höchst bemerkenswerth ist noch meines Erachtens jener bereits oben erwähnte Umstand, dass grob makroskopische, sogenannte Hemmungs- 
bildungen in keiner der zwei Formen vorkommen; beide sind ausschliesslich mikroskopisch, eytopathologisch charakterisirt in der Form einer Veränderung, welche sich auf das gesammte Centralgrau erstreckt.

Auf Grund dieser Analyse fühle ich mich zu folgendem Schluss gedrängt. Spielmayer's juvenile Form der familiär-amaurotischen Idiotie hat dasselbe histopathologische Substrat wie die Sachs'scbe infantile Form, mit der Bemerlzung, dass letztere bezüglich der Intensität der Zellentartung eine entschieden schwerere Form darstellt. Somit verificire ich auf Grund anatomisch-histologischer Untersuchung H. Vogt's Aufstellung bezüglich eines grossen einheitlichen Typus von familiäramaurotischer Idiotie: H. Vogt hat das Richtige getroffen, als er auf Grund klinischer Betrachtungen die Sachs'schen Fälle als infantile Form mit der juvenilen Form in einen einheitlichen Typus zusammenfasste und die Sachs'sche Gruppe als eine intensivere Form auffasste, denn sie setzt in früherem Alter ein "das Gehirn versagt rascher" und verläuft auch im schnelleren Tempo, mehr foudroyant.

Es will mich bedünken, dass Angesichts dieses Resultates der anatomischen Forschung die oben angeführten klinischen Differenzen, in allererster Reihe die Veränderungen des Augenhintergrundes, trennende llomente von secundärer, klinisch-classificatorischer Bedeutung sind. Ich bin daher der Ansicht, dass es eine grosse, einheitliche Form von familiär-amaurotischer ldiotie giebt, welche zwar verschiedene klinische Varietäten in sich birgt, jedoch morphologisch durch gewisse Uebereinstimmung der allgemeinen Züge als eine klinische Familie charakterisirt ist. So dürfte es eine grosse Idiotieform geben, welche rein cellularpathologisch gekennzeichnet ist, namentlich durch die mehr minder ausgeprägte Schwellung des Zellleibes sowie der Dendriten; ein besonderer morphologischer Charakterzug dieser grossen Idiotieform wäre ferner noch die absolute Diffusion der Zellerkrankung auf das gesammte Centralgrau nebst fehlenden makroskopischen Anomalien. Hierher wäre dann die schwerere Sachs'sche und die leichtere Spielmayer'sche Form zu reihen als zwei Glieder der grossen klinischen Familie; welche wir die eytopathologisch charakterisirte familiär-amaurotische Idiotie nennen könnten. Doch giebt es, wie mein vorliegender Fall beweist, noch eine teratologisch begründete Form der amaurotischen Idiotie, welcher aber - wie dies unschwer vorauszusehen ist - der familiäre Charakter abgehen dürfte, somit hat diese mit der familiär-amaurotischen Idiotie nichts Gemeinsames. 